\title{
Overlap functions, spectroscopic factors, and asymptotic normalization coefficients generated by a shell-model source term
}

\author{
N. K. Timofeyuk \\ Department of Physics, University of Surrey, Guildford, Surrey GU2 7XH, UK
}

(Received 16 March 2010; published 4 June 2010)

\begin{abstract}
Overlap functions for one-nucleon removal are calculated as solutions of the inhomogeneous equation. The source term for this equation is generated by the $0 \hbar \omega$ no-core shell-model wave functions and the effective nucleon-nucleon $(N N)$ interactions that fit oscillator matrix elements derived from the $N N$ scattering data. For the lightest $A \leqslant 4$ nuclei this method gives reasonable agreement with exact $a b$ initio calculations. For $4<A \leqslant 16$, this method gives a fair agreement between the calculated and measured asymptotic normalization coefficients. The spectroscopic factors obtained show systematic deviation from the corresponding shell-model values. This deviation correlates with nucleon separation energies and follows a similar trend seen in the reduction factor of the nucleon knockout cross sections. Comparison with the overlap functions and spectroscopic factors obtained in the variational Monte Carlo method is presented and discussed.
\end{abstract}

DOI: 10.1103/PhysRevC.81.064306

PACS number(s): 21.10.Jx, 27.20.+n, 27.10.+h

\section{INTRODUCTION}

The spectroscopic factors (SFs), introduced over fifty years ago in the theory of transfer reactions to link nuclear reactions and structure, are usually associated with occupancies of single-particle orbits. It has been widely believed that experimental study of SFs provides an important tool to explore single-particle structure of nuclei. In the vast literature published over this period, the experimental SFs $S_{\text {exp }}$, determined mostly from transfer reactions as ratios of experimental to theoretical cross sections, were compared to theoretical predictions, $S_{\text {th }}$, mainly from the shell model. In many publications $S_{\exp }$ and $S_{\text {th }}$ were often in reasonable agreement. It has been found, however, that proton SFs in the closed-shell nuclei ${ }^{16} \mathrm{O},{ }^{40,48} \mathrm{Ca}$, and ${ }^{208} \mathrm{~Pb}$, obtained from $\left(e, e^{\prime} p\right)$ reactions, the mechanism of which is much better understood than that of transfer reactions, are 50\%-60\% smaller than those expected from the independent-particle model [1]. A similar reduction of SFs has been found for other nuclei such as ${ }^{7} \mathrm{Li},{ }^{12} \mathrm{C},{ }^{30} \mathrm{Si},{ }^{31} \mathrm{P},{ }^{51} \mathrm{~V}$, and ${ }^{90} \mathrm{Zr}$ [2]. The apparent discrepancy between SFs obtained from transfer and $\left(e, e^{\prime} p\right)$ reactions has been shown to originate from the different bound-state wave functions $\varphi(r)$ employed in the analysis of these reactions [2]. The $\left(e, e^{\prime} p\right)$ reaction is sensitive to the whole $\varphi(r)$ whereas transfer reactions are only sensitive to $\varphi(r)$ at large $r$, where the radial form is fixed by the nucleon separation energy while the magnitude depends strongly on the assumed shape of the potential well used to generate $\varphi(r)$. Employing $\varphi(r)$, derived from the $\left(e, e^{\prime} p\right)$ momentum distributions, in transfer reactions brings their SFs into close agreement with those obtained from $\left(e, e^{\prime} p\right)$ reactions, thus confirming the $\mathrm{SF}$ reduction. In a recent analysis of the $(d, p)$ and $(p, d)$ reactions [3], where $\varphi(r)$ is fixed by modern Hartree-Fock calculations and has a shape similar to that derived from $\left(e, e^{\prime} p\right)$, reduced SF values have been reported. The SF reduction has also been observed in high-energy nucleon removal reactions from ${ }^{12} \mathrm{C}$ and ${ }^{16} \mathrm{O}$ [4].

With the development of radioactive beam facilities SF studies have been extended to nuclei away from the valley of stability. A new phenomenon has been revealed with the help of one-nucleon knockout reactions. It turns out that reduction of SFs from the shell-model values depends on the separation energy of the removed nucleon and on nucleon type and that the SF reduction factor, defined as $R_{s}=S_{\text {exp }} / S_{\text {th }}$, is concentrated around a straight line when plotted as a function of the difference between proton $\left(S_{p}\right)$ and neutron $\left(S_{n}\right)$ separation energies, $\Delta S$, taken as $S_{p}-S_{n}$ and $S_{n}-S_{p}$ for proton and neutron knockout, respectively [5].

The $R_{s}(\Delta S)$ dependence has been assumed to arise from correlations missing from the truncated shell-model space [5]. Increasing the model space should then reduce the gap between $S_{\text {th }}$ and $S_{\text {exp }}$. Indeed, in a six-shell treatment of ${ }^{16} \mathrm{O}$ the percentage of the $0 \hbar \omega$ component goes down to $\sim 48 \%-60 \%$ [6] and the ${ }^{16} \mathrm{O}$ SF decreases from the $0 \hbar \omega$ value of 2 to 1.65 [4]. However, the six-shell model space is still not sufficient to reproduce the $\left(e, e^{\prime} p\right)$ value of 1.27(13) [2]. The necessity of adding more major shells to the model space implies that the common view of ${ }^{16} \mathrm{O}$ as a doubly magic nucleus is not correct.

The contributions from missing model space can be recovered by using correlated wave functions in $a b$ initio approaches. Thus, the variational Monte Carlo (VMC) calculations for ${ }^{7} \mathrm{Li}$ result in a proton $\mathrm{SF}$ that is reduced from the $0 \hbar \omega$ value by $40 \%$ but agrees very well with $S_{\text {exp }}$ from $\left(e, e^{\prime} p\right)$ [7]. However, for ${ }^{8,9} \mathrm{Li},{ }^{8} \mathrm{~B}$, and ${ }^{9} \mathrm{C}$ the VMC SFs are larger than $S_{\text {exp }}$ (see Table VII). Also, the $a b$ initio VMC calculations are feasible only for light nuclei while the SF reduction is observed for nuclei as heavy as ${ }^{208} \mathrm{~Pb}$. In the medium- and heavy-mass regions, different approaches are being developed to deal with correlations beyond the shell-model space. For example, a correlated basis function theory [8] can account for short-range correlations, related to the strongly repulsive core of the nucleon-nucleon $(N N)$ interaction. However, the SF reduction obtained within this theory for the closed-shell nuclei ${ }^{16} \mathrm{O},{ }^{40} \mathrm{Ca},{ }^{48} \mathrm{Ca}$, and ${ }^{208} \mathrm{~Pb}$ does not exceed $15 \%$, which is inconsistent with experimental data. Long-range correlations, associated with quasiparticle excitations, can be taken into 
account within the self-consistent Green's function method [9]. The SFs obtained within this method for ${ }^{48} \mathrm{Ca}$ and ${ }^{56} \mathrm{Ni}$ are in agreement with those obtained from $\left(e, e^{\prime} p\right)$ and heavyion knockout experiments, thus showing that the long-range correlations in SF reduction are much more important than the short-range ones. However, a more systematic application of this method to various closed-shell nuclei ranging from ${ }^{16} \mathrm{O}$ to ${ }^{60} \mathrm{Ca}$ shows that reduction factors cannot be smaller than 0.73 [10]. It should be mentioned again that approaches similar to the correlated basis function method and the self-consistent Green's function method can be applied only to those nuclei that are traditionally considered as the closed-shell ones. It is too difficult to apply such approaches to open-shell nuclei with many valence nucleons. Therefore, for many nuclei, the standard shell-model SF calculations will still be used for a long time.

In my previous publication [11], it was shown that it is legitimate to use uncorrelated wave functions defined in minimal model spaces for the SF calculations provided the overlap function, the norm of which gives the SF, is obtained as a solution of an inhomogeneous equation. In this procedure, the SFs obtained depend strongly on the effective $N N$ interactions used to calculate the source term that generates the overlap functions. It was shown that an effective two-body $N N$ potential exists that gives reasonable predictions for overlap functions of the deuteron, ${ }^{3,4} \mathrm{He}$, and ${ }^{16} \mathrm{O}$ and at the same time reproduces fairly the $R_{S}(\Delta S)$ dependence for the ground states of the $0 p$-shell nuclei. In the present paper, I give the details of the inhomogeneous equation approach missing in Ref. [11] and discuss the outcome of calculations in more detail. First, I discuss in Sec. II the problems of the standard shell-model overlap calculations. Then, in Sec. III, the inhomogeneous equation method is presented and discussed. The results for $A \leqslant 4$ and $4 \leqslant A \leqslant 16$ are given in Secs. IV and V, respectively. The energy dependence of SFs in discussed in Sec. VI and Sec. VII presents a comparison between the present and $a b$ initio calculations. Conclusions are presented in Sec. VIII and some details of the fractional-parentage technique used to calculate the necessary matrix elements are given in an appendix.

\section{CALCULATING THE OVERLAP FUNCTIONS BY DIRECT EVALUATION OF THE OVERLAP INTEGRAL}

The theoretical SF for one-nucleon removal, $S_{l j}$, is defined in a model-independent way as

$$
S_{l j}=\int_{0}^{\infty} d r r^{2} I_{l j}^{2}(r),
$$

where $I_{l j}(r)$ is the radial overlap function with orbital momentum $l$ and angular momentum $j$, calculated as an overlap integral between the wave functions $\Psi_{J_{B}}$ and $\Psi_{J_{A}}$ of two neighboring nuclei $B=A-1$ and $A$ with the total spin $J_{B}$ and $J_{A}$ :

$$
I_{l j}(r)=A^{1 / 2}\left\langle\left[\left[Y_{l}(\hat{\boldsymbol{r}}) \otimes \chi_{1 / 2}^{\tau}\right]_{j} \otimes \Psi_{J_{B}}\right]_{J_{A}} \mid \Psi_{J_{A}}\right\rangle .
$$

Here the integration is carried out over $3 A-6$ independent coordinates describing the internal structure of nucleus $B$, $r$ is the distance between the center of mass of $B$ and the removed nucleon, $Y_{l}$ is the spherical function, and $\chi_{1 / 2}^{\tau}$ is the spin-isospin function of the removed nucleon with isospin projection $\tau$. The coefficient $A^{1 / 2}$ comes from antisymmetrization. If not included in Eq. (2), it should appear in the definition [Eq. (1)] of the SF.

All available phenomenological shell-model codes calculate $S_{l j}$ from $I_{l j}(r)$ obtained by direct evaluation of Eq. (2) using some model wave functions in truncated model spaces presented as linear combinations of Slater determinants made of the single-particle functions $\psi_{\alpha}(\boldsymbol{r})$. The only quantities that matter in the phenomenological shell model are the matrix elements of the effective nucleon-nucleon $(N N)$ interaction, $\left\langle\psi_{\alpha_{1}}\left(\boldsymbol{r}_{1}\right) \psi_{\alpha_{2}}\left(\boldsymbol{r}_{2}\right)\left|v^{\text {eff }}\left(r_{12}\right)\right| \psi_{\alpha_{3}}\left(\boldsymbol{r}_{1}\right) \psi_{\alpha_{4}}\left(\boldsymbol{r}_{2}\right)\right\rangle$, and the single-particle energies. The matrix elements are just the sets of numbers fitted to a range of nuclear spectra, and they do not contain information about the radial shapes of the $\psi_{\alpha}(\boldsymbol{r})$. To calculate SFs, in the shell model it is assumed that the single-particle wave functions have the same shapes in nuclei $B$ and $A$. In this case, the shape of $\psi_{\alpha}(\boldsymbol{r})$ are not needed because the only property that is used in the calculations is the orthogonality of the single-particle basis, $\left\langle\psi_{\alpha_{1}}(\boldsymbol{r}) \mid \psi_{\alpha_{2}}(\boldsymbol{r})\right\rangle=$ $\delta_{\alpha_{1} \alpha_{2}}$. The resulting SFs are related only to the occupancies of the single-particle states in two neighboring nuclei, determined by the shell-model $N N$ matrix elements. The situation is even simpler for the overlap functions involving closed-shell nuclei; their SFs do not depend on the effective interactions at all.

The phenomenological shell model employs wave functions that are not translationally invariant. However, if the singleparticle wave functions are assumed to be generated by the oscillator potential well then the center-of-mass (c.m.) correction to SFs in the $0 \hbar \omega$ model space is easily obtained. It is equal to $[A /(A-1)]^{n}$, where $n$ is the number of oscillator quanta carried away by the removed nucleon. For example, the $0 \hbar \omega \mathrm{SF}$ of ${ }^{16} \mathrm{O}$ corrected for the c.m. motion is 2.133 , which increases the gap between the $0 \hbar \omega$ shell model and the $\left(e, e^{\prime} p\right)$ values. No c.m. corrections are available for other shapes of $\psi_{\alpha}(\boldsymbol{r})$.

The knowledge of SFs alone is insufficient for predicting one-nucleon removal cross sections where the radial shapes of $I_{l j}(r)$ are crucial. In most applications, these shapes are found from the separation-energy prescription [12], not related to the shell-model $N N$ matrix elements. This prescription involves fitting the depth of the potential well, used to generate $\psi_{\alpha}(\boldsymbol{r})$, to reproduce the experimental separation energy of the removed nucleon. Sometimes, for example in Ref. [3], it is assumed that the shape of $I_{l j}(r)$ is the same as the shape of $\psi_{\alpha}(\boldsymbol{r})$ obtained by the Hartree-Fock calculations. In these cases, the $I_{l j}(r)$ shape is related to the effective $N N$ interactions but its norm is not since in Hartree-Fock theory one assumes independent particle motion.

The common problem for both the shell model and HartreeFock theory is that their wave functions span the allowed model space $P$ only. The contributions from the missing space $Q$ are taken into account only for the binding energy via effective $N N$ interactions. No terms of the $\left\langle\Psi_{P}(A-1) \mid \Psi_{Q}(A)\right\rangle$, $\left\langle\Psi_{Q}(A-1) \mid \Psi_{P}(A)\right\rangle$, or $\left\langle\Psi_{Q}(A-1) \mid \Psi_{Q}(A)\right\rangle$ types are ever considered when overlap functions are calculated. The same is true about the ab initio no-core shell-model calculations in 
which effective $N N$ interactions are constructed from realistic ones. Including the contributions from $Q$ can be achieved if an exact nuclear wave function $\Psi$ is constructed from an uncorrelated state $\Phi$, defined in some truncated model space $P$, by acting on it by an operator. There are several ways of choosing such an operator. A convenient one is to use the unitary correlation operator method (UCOM) [13], according to which

$$
|\Psi\rangle=C|\Phi\rangle=\exp \left\{-i \sum_{i<j}^{A} g_{i j}\right\}|\Phi\rangle .
$$

Here $C$ is the unitary correlator designed to shift nucleons away from each other whenever their uncorrelated positions are within the repulsive $N N$ core. In the UCOM, $\Phi$ is found from an effective Hamiltonian that contains an effective interaction $V^{\text {eff }}$ consisting of $\hat{V}=C^{\dagger} V C$ and the terms arising from the kinetic-energy operator [13]. If wave functions from Eq. (3) are used in Eq. (2), then

$$
\left\langle\Psi_{B} \mid \Psi_{A}\right\rangle=\left\langle\Phi_{B}\left|C_{B}^{\dagger} C_{A}\right| \Phi_{A}\right\rangle=\left\langle\Phi_{B}\left|C_{N B}\right| \Phi_{A}\right\rangle,
$$

where $C_{A}=C_{B} C_{N B}$ and $C_{N B}=\exp \left\{-i \sum_{i=1}^{B} g_{i N}\right\}$, under the assumption that unitary correlators in $B$ and $A$ are the same. As shown in Ref. [13], this assumption is reasonable if the space $P$ is formed by the fermionic molecular dynamic model wave functions. Equation (4) shows that, to include contributions from missing model spaces, the matrix elements of the operator $C_{N B}$ have to be calculated. This operator plays the role of a renormalized one-nucleon removal operator. In the shell model, the Hartree-Fock model, and many other microscopic methods one assumes that

$$
\left\langle\Psi_{B} \mid \Psi_{A}\right\rangle \approx\left\langle\Phi_{B} \mid \Phi_{A}\right\rangle .
$$

In other words, no renormalization is done for the nucleon removal operator.

One important property of the overlap function is its asymptotic behavior,

$$
I_{l j}(r) \approx C_{l j} \frac{W_{-\eta, l+1 / 2}(2 \kappa r)}{r}, \quad r \rightarrow \infty,
$$

where $\eta=Z_{B} Z_{N} e^{2} \mu / \hbar^{2} \kappa, Z_{B}$ and $Z_{N}$ are the charge of $B$ and of the removed nucleon $N$, respectively, $\kappa=\left(2 \mu \varepsilon / \hbar^{2}\right)^{1 / 2}$, $\varepsilon=E_{A}-E_{B}, E_{A}$ and $E_{B}$ are the binding energies of nuclei $A$ and $B$, respectively, $\mu$ is the reduced mass, $W$ is the Whittaker function, and $C_{l j}$ is the asymptotic normalization coefficient (ANC). It is this part of the overlap function that often determines the angular distributions of transfer reactions and is important for all other nucleon removal reactions. The phenomenological shell model does not have single-particle wave functions and therefore it cannot provide the behavior given by Eq. (6), nor can other microscopic approaches guarantee this behavior unless the single-particle wave functions are included explicitly in the model wave functions. This can be achieved by using cluster-type models combined with the $R$-matrix approach as, for example, in Ref. [14], or by tuning the mean-field potentials to get the desired rate of decrease of the single-particle states at large $r$, as in Ref. [15]. Using Eq. (4) may not be helpful to obtain the proper asymptotic behavior. Indeed, the short-range correlators influence mostly those parts of the wave functions where two nucleons are close to each other. As a consequence, only the internal part of the overlap functions will be affected by the short-range correlations while the asymptotic part will remain unaffected and contain no information about the excluded model space. Increasing the model space $P$ is therefore crucial for the asymptotic part of $I_{l j}(r)$.

\section{OVERLAP INTEGRALS AND THE INHOMOGENEOUS EQUATION}

It has been shown by Pinkston and Satchler [16] that the overlap function $I_{l j}(r)$ is a solution of the inhomogeneous equation (IE)

$$
\left\langle\Psi_{B}\left|\hat{T}_{A}-\hat{T}_{B}-E_{A}+E_{B}\right| \Psi_{A}\right\rangle=\left\langle\Psi_{B}\left|V_{B}-V_{A}\right| \Psi_{A}\right\rangle,
$$

in which the right-hand side (r.h.s.) (the source term) can be treated as known. In Eq. (7), $\hat{T}_{i}$ and $V_{i}$ are the kinetic- and potential-energy operators of nucleus $i$. Equation (7) generates an $I_{l j}(r)$ that automatically has the correct asymptotic shape when the experimental value of $\varepsilon$ is used, whatever $\Psi_{J_{A}}$ and $\Psi_{J_{B}}$ are

Earlier explorations of the inhomogeneous equation method were carried out in the 1960s-1970s. A mean-field picture of nuclei was assumed, in which the potential $V$ was decomposed into a mean-field part and a residual interaction (see, for example, Ref. [17]). The mean-field part was removed from the source term on the left-hand side (1.h.s.) of Eq. (7), leaving in the r.h.s. residual interactions only. The wave functions $\Psi_{A}$ and $\Psi_{B}$ were represented by uncorrelated products of single-particle functions in the valence-nucleon model space under the assumption of configuration mixing. $\Psi_{A}$ and $\Psi_{B}$ were obtained by diagonalizing a Hamiltonian that contains the same residual interactions used to calculate the source term, in this valence nucleon space. Also, the requirement that the derived spectroscopic amplitudes should agree with the corresponding shell-model amplitudes, obtained through a direct evaluation of $I_{l j}(r)$, was imposed as a condition on the residual potentials employed. Their choices were important for the shape of $I_{l j}(r)$ and, therefore, for the determination of $S_{\text {exp }}$ through the comparison of theoretical cross sections, calculated with this $I_{l j}(r)$, to the experimental ones. A review of all early explorations using Eq. (7), presented in Ref. [12], concludes that these efforts gave little information about the utility of the method. They were abandoned before the 1980s.

An alternative way to calculate $I_{l j}(r)$ for one-neutron removal has been developed in Refs. [18,19]. It is based on the relation between the Fourier transform $I_{l j}(q)$ of this overlap function and the vertex form factor $G_{l j}(q)$ that appears in the dispersion theory of nuclear reactions [20], namely

$$
I_{l j}(q)=-4 \mu \sqrt{\pi} G_{l j}(q) /\left(\kappa^{2}+q^{2}\right) .
$$

The vertex form factor is in turn a Fourier transform of the same source term that appears in Eq. (7),

$$
G_{l j}(q)=\sqrt{4 \pi}\left\langle\left[\left[j_{l}(q r) Y_{l}(\hat{\boldsymbol{r}}) \otimes \chi_{\frac{1}{2}}^{\tau}\right]_{j} \otimes \Psi_{J_{B}}\right]_{J_{A}}\left\|V_{N B}\right\| \Psi_{J_{A}}\right\rangle,
$$


where $V_{N B}=\sum_{i=1}^{B} v_{i A}$. The vertex form factor taken at $q=$ $i \kappa$, called the vertex constant, has the same meaning as the coupling constants in particle physics and it is related by a simple equation to the ANC $C_{l j}$, namely

$$
G_{l j} \equiv G_{l j}(i \kappa)=-\sqrt{\pi}(\hbar / \mu c) C_{l j} .
$$

The original motivation of the vertex form factor study, performed in Refs. [18,21], was to provide a theoretical calculation for the vertex constants extracted from transfer reactions on light nuclei using a range of methods [22]. A continuation of this work in Ref. [19] has provided SFs as well. For this purpose, $I_{l j}(r)$ has been obtained from $I_{l j}(q)$ as an inverse Fourier transform and then its norm has been evaluated. Such a procedure is equivalent to solving the inhomogeneous equation (7) in momentum space. The source term arising in Eq. (9) has been calculated in Refs. [18,19,21] in which it was assumed that the wave functions $\Psi_{J_{A}}$ and $\Psi_{J_{B}}$ could be approximated by the no-core oscillator $0 \hbar \omega$ shell-model wave functions $\Phi_{A}$ and $\Phi_{B}$. Various effective two-body $v_{i A}$ potentials were used from those available in the literature. It was found that a reasonable agreement between measured and calculated vertex constants is achieved if a version of the M3Y potential, constructed in Ref. [23] to fit the oscillator matrix elements derived from the $N N$ scattering phase shifts, is used for $V_{N B}$ in Eq. (9). However, at the same time the calculated SFs could differ strongly from the original shell-model values obtained from $I_{l j}(r)$ calculated using Eq. (4). This result was not understood at the time. It was suggested that these discrepancies originated because the source term was calculated using $v_{i A}$, which was different from that used to diagonalize the shell-model Hamiltonian. A test study of ${ }^{8} \mathrm{~B}$, performed later in ref. [24], has shown that even if the same interaction were employed, the difference between SFs obtained using Eq. (4) and Eq. (7) can still be large. However, the predicted vertex constants were much closer to the experimental values if $v_{i A}$ was different from the one used to diagonalize the shell-model Hamiltonians.

In all previous publications employing the idea of the source term, no consideration has been given to the consequence of restricting the model space on its calculation. Prior to discussing this issue, let me note that the exact solution $I_{l j}(r)$ of the inhomogeneous equation (7) can be written as [24]

$$
\left.I_{l j}(r)=A^{\frac{1}{2}} /\left[\left[\frac{G_{l}\left(r, r^{\prime}\right)}{r r^{\prime}} Y_{l}\left(\hat{\boldsymbol{r}^{\prime}}\right) \otimes \chi_{\frac{1}{2}}^{\tau}\right]_{j} \otimes \Psi_{J_{B}}\right]_{J_{A}}\|\hat{\mathcal{V}}\| \Psi_{J_{A}}\right\rangle,
$$

where integration over $r^{\prime}$ is implied and $G_{l}\left(r, r^{\prime}\right)$, the Green's function for a bound nucleon in the field of a point charge $Z_{B}$, is

$$
G_{l}\left(r, r^{\prime}\right)=-\frac{2 \mu}{\hbar^{2} \kappa} e^{-\pi i(l+1+\eta) / 2} F_{l}\left(i \kappa r_{<}\right) W_{-\eta, l+1 / 2}\left(2 \kappa r_{>}\right),
$$

corresponding to the momentum $i \kappa$. Here $F$ is the regular Coulomb function. Also, $\hat{\mathcal{V}}=V_{A}-V_{B}-V_{c}^{0}, V_{x}=\sum_{i<j}^{x} v_{i j}$, and $V_{c}^{0}$ is the point Coulomb interaction between the valence nucleon and $B$. In the following, the overlap function obtained as a solution of the inhomogeneous equation is denoted as $I_{l j}^{\mathrm{IE}}(r)$ and the overlap function obtained by direct evaluation of the integral (2) is called $I_{l j}^{\mathrm{DE}}(r)$.

Equation (11) has been obtained by assuming that $\Psi_{J_{B}}$ and $\Psi_{J_{A}}$ are exact solutions of the many-body Schrödinger equation and that $\hat{\mathcal{V}}$ contains bare realistic $N N$ interactions, in which case $I_{l j}^{\mathrm{IE}}(r)$ should be exactly equal to $I_{l j}^{\mathrm{DE}}(r)$. However, the approximations $\Psi_{J_{B}} \approx \Phi_{B}$ and $\Psi_{J_{A}} \approx \Phi_{A}$ require renormalization of one-nucleon removal operators in Eqs. (2) and (11). In both cases this renormalization leads to many-body operators, evaluation of which requires approximations to be made. The approximations can play different roles in Eqs. (2) and (11), which can give rise to the inequality $I_{l j}^{\mathrm{IE}}(r) \neq I_{l j}^{\mathrm{DE}}(r)$. Therefore, the choice of the interaction $V_{N B}$ based on the requirement of $S_{l j}^{\mathrm{IE}}=S_{l j}^{\mathrm{DE}}$, where $S_{l j}^{\mathrm{IE}}$ and $S_{l j}^{\mathrm{DE}}$ are the $\mathrm{SFs}$ corresponding to $I_{l j}^{\mathrm{IE}}(r)$ and $I_{l j}^{\mathrm{DE}}(r)$, is not justified.

The renormalization for $\hat{\mathcal{V}}$ should depend on the particular method of connecting the truncated and excluded model spaces. A simple way to get it for $\hat{\mathcal{V}}$ is to consider the UCOM wave functions given by Eq. (3) and to use them in Eq. (11). This gives

$$
\begin{aligned}
\left\langle\Psi_{B}|\hat{\mathcal{V}}| \Psi_{A}\right\rangle & =\left\langle\Phi_{B}\left|C_{B}^{\dagger}\left(V_{A}-V_{B}\right) C_{A}\right| \Phi_{A}\right\rangle \\
& =\left\langle\Phi_{B}\left|V_{N} C_{N B}\right| \Phi_{A}\right\rangle=\left\langle\Phi_{B}\left|\tilde{V}^{\mathrm{eff}}\right| \Phi_{A}\right\rangle .
\end{aligned}
$$

One can see that the effective interaction $\tilde{V}^{\text {eff }}$, which replaces $\hat{\mathcal{V}}$ when modeling the source term in the truncated model space, differs from the effective interactions $V_{B}^{\text {eff }}$ and $V_{A}^{\text {eff }}$ that generate $\Phi_{B}$ and $\Phi_{A} . V^{\text {eff }}$ includes two correlators, as it has $C^{\dagger} V C$, and the terms arising from the kinetic energy operator, whereas $\tilde{V}^{\text {eff }}$ has only one correlator and no terms from the kinetic energy. On this basis, it is not justified to use the same effective interaction both in calculations of $\Phi$ and for $\tilde{V}^{\text {eff }}$ as assumed in earlier studies of the Pinkston-Satchler type.

Equation (13) tells us that the source term and therefore the corresponding $I_{l j}^{\mathrm{IE}}(r)$ and $S_{l j}^{\mathrm{IE}}$ depend on those components of the $N N$ interactions to which the binding-energy calculations are not sensitive at all. This feature of the overlaps has not been appreciated before as it cannot be noticed if the overlaps and SFs are calculated in the usual way using the approximation of Eq. (5). To demonstrate this, let us keep only two-body terms in the operator $\tilde{V}^{\text {eff }}$, neglect the center-of-mass motion, and assume that $\psi_{\alpha}$ are generated by the oscillator shell model. Then the following matrix elements determine $I_{l j}^{\mathrm{IE}}(r):\left\langle G_{l}\left(r, r_{1}\right) /\left(r_{1} r\right) \psi_{\alpha_{2}}\left(\boldsymbol{r}_{2}\right)\left|\tilde{v}^{\mathrm{eff}}\left(r_{12}\right)\right| \psi_{\alpha_{3}}\left(\boldsymbol{r}_{1}\right) \psi_{\alpha_{4}}\left(\boldsymbol{r}_{2}\right)\right\rangle$ or $\left\langle j_{l}\left(q r_{1}\right) \psi_{\alpha_{2}}\left(\boldsymbol{r}_{2}\right)\left|\tilde{v}^{\text {eff }}\left(r_{12}\right)\right| \psi_{\alpha_{3}}\left(\boldsymbol{r}_{1}\right) \psi_{\alpha_{4}}\left(\boldsymbol{r}_{2}\right)\right\rangle$, depending on whether the coordinate or momentum representation for the overlap function is used. Both $G_{l}\left(r_{1}, r^{\prime}\right)$ and $j_{l}(q r)$ can be expanded onto the single-particle basis $\psi_{\alpha}$. Because the contribution to the matrix elements comes from an $r$ that is 2-3 times larger than the typical values of the oscillator radius, the $I_{l j}^{\mathrm{IE}}(r)$ will contain a contribution from a large (possibly infinite) number of $\psi_{\alpha}$ from the model space absent in $\Phi_{A}$ or $\Phi_{B}$. Thus, the very use of the inhomogeneous equation guarantees recovering the missing contributions from the excluded model spaces.

It may appear that renormalizing the one-nucleon removal operator, in other words, using Eq. (4), should recover 
contributions from the missing spaces as well. However, as discussed in the previous section, it hardly influences the asymptotic region of $I_{l j}(r)$ and it is likely to be inefficient in the nuclear interior as well. Indeed, by retaining the two-body terms of the correlator $C_{N B}$, the $S_{l j}^{\mathrm{DE}}$ should contain a contribution of the following type:

$$
\begin{array}{r}
\int d \boldsymbol{r} \int d \boldsymbol{r}_{1} \psi_{\alpha_{1}}\left(\boldsymbol{r}_{1}\right) g^{\dagger}\left(\boldsymbol{r}_{1}-\boldsymbol{r}\right) \psi_{\alpha_{2}}^{\dagger}\left(\boldsymbol{r}_{1}\right) \psi_{\alpha_{3}}^{\dagger}(\boldsymbol{r}) \\
\times \int d \boldsymbol{r}_{2} \psi_{\alpha_{1}^{\prime}}\left(\boldsymbol{r}_{2}\right) g\left(\boldsymbol{r}_{2}-\boldsymbol{r}\right) \psi_{\alpha_{2}^{\prime}}\left(\boldsymbol{r}_{2}\right) \psi_{\alpha_{3}^{\prime}}(\boldsymbol{r}),
\end{array}
$$

provided the center-of-mass motion is excluded. Changing the integration variables $\left\{\boldsymbol{r}, \boldsymbol{r}_{1}, \boldsymbol{r}_{2}\right)$ to $\left\{\boldsymbol{r}_{1}-\boldsymbol{r}, \boldsymbol{r}_{2}-\boldsymbol{r},\left(\boldsymbol{r}+\boldsymbol{r}_{1}+\right.\right.$ $\left.\left.\boldsymbol{r}_{2}\right) / 3\right\}$ and expanding the oscilator wave functions $\psi_{\alpha}(\boldsymbol{r})$ over the basis functions in new coordinates, one obtains that the SFs should contain the following contributions:

$$
\left(\int d \boldsymbol{r} \psi_{\beta_{1}^{\prime}}^{\dagger}(\boldsymbol{r}) g^{\dagger}(\boldsymbol{r}) \psi_{\beta_{2}^{\prime}}(\boldsymbol{r})\right) \cdot\left(\int d \boldsymbol{r} \psi_{\beta_{1}}^{\dagger}(\boldsymbol{r}) g(\boldsymbol{r}) \psi_{\beta_{2}}(\boldsymbol{r})\right)
$$

in which the sum of the oscillator quanta $N$ satisfies $N_{\beta_{1}}+N_{\beta_{2}}+N_{\beta_{1}^{\prime}}+N_{\beta_{2}^{\prime}} \leqslant N_{\alpha_{1}}+N_{\alpha_{2}}+N_{\alpha_{3}}+N_{\alpha_{1}^{\prime}}+$ $N_{\alpha_{2}^{\prime}}+N_{\alpha_{3}^{\prime}}$. Thus, if each oscillator orbital in the allowed model space has no more than $N$ oscillator quanta then the highest oscillator shell of the excluded space connected by the correlator $g(\boldsymbol{r})$ is $N+6$; in other words, the renormalization of the one-nucleon operators in the direct evaluation of overlap integrals has a restricted influence on the spectroscopic factors. This is implicitly confirmed by the correlated-basis calculations in Ref. [8], where reduction of the spectroscopic factors for the closed shell due to the short-range correlations is no more than $15 \%$.

Although the application of the inhomogeneous equation promises to solve efficiently the problem of excluded states, the question remains how to construct correctly the $\tilde{V}^{\text {eff }}$. There are two ways to deal with this problem: either to calculate it using one or another microscopic theory or to treat it phenomenologically. The phenomenological approach has in fact been used in Refs. [18,19,21,24]. What has been done there is equivalent to the following implicit assumptions: The correlators are the same in two neighboring nuclei, the contributions from both the three-body force and the three-body and higher terms coming from expansion of correlators are negligible, and the effective interactions are local. The quantity that served in Refs. $[18,21]$ as a reference to calibrate $\tilde{V}^{\text {eff }}$ was the vertex constant. Since this constant is trivially related by Eq. (10) to the ANC, this is equivalent to choosing ANCs as a reference for calibrating $\tilde{V}^{\text {eff }}$. The ANCs depend on the same source term as the overlap functions and spectroscopic factors and they can be more easily determined from peripheral transfer reactions than SFs, provided that the reaction theories used for these purposes are adequate. In the following, I present new phenomenological calculations of overlap functions, SFs, and ANCs for $0 p$-shell nuclei. There are two reasons for new calculations: (i) In old calculations some small terms of the wave functions were neglected and this could lead to some inaccuracies of the results obtained and (ii) improved phenomenological interactions that fit better the spectra of $0 p$-shell nuclei are available today and can help to fix the allowed model space in a better way.

\section{SHELL-MODEL CALCULATIONS FOR NUCLEI WITH THE SIMPLEST STRUCTURE}

In this section, the overlap functions $\langle A-1 \mid A\rangle$ for nuclei that can be described by only one Slater determinant, such as ${ }^{3} \mathrm{H},{ }^{3,4} \mathrm{He}$, and ${ }^{16} \mathrm{O}$, are considered. For these nuclei, the overlap functions, spectroscopic factors, and ANCs do not depend on effective two-body interactions that fit nuclear spectra. This gives us an opportunity to understand $\tilde{V}^{\text {eff }}$ better.

\section{A. $A=2$}

First, the method is applied to the well-understood $A=2$ system, for which $I_{01 / 2}^{\mathrm{IE}}(r)$ is the radial deuteron wave function of the $s$-wave motion. Equation (11) becomes in this case

$$
r I_{01 / 2}^{\mathrm{IE}}(r)=\int_{0}^{\infty} d r^{\prime} r^{\prime} G_{0}\left(r, r^{\prime}\right) \tilde{V}^{\mathrm{eff}}\left(r^{\prime}\right) \varphi_{0 s}\left(r^{\prime}\right),
$$

where

$$
\varphi_{0 s}(r)=\frac{2}{r_{0}^{3 / 2} \pi^{1 / 4}} \exp \left(-\frac{r^{2}}{2 r_{0}^{2}}\right)
$$

is the $0 s$ oscillator wave function with oscillator radial parameter $r_{0}$. It was found that for a fixed potential $\tilde{V}^{\text {eff }}\left(r^{\prime}\right)$ the choice of $r_{0}$ does not influence much the shape of $I_{01 / 2}^{\mathrm{IE}}(r)$. However, it does influence its norm. This is demonstrated in Fig. 1(a), which shows the ANCs, the SFs (the norms), and the rms radii of $I_{01 / 2}^{\mathrm{IE}}(r)$, calculated with the M3YE potential, as a function of $r_{0}$ chosen in a range from 1.3 to $1.7 \mathrm{fm}$ typical for the lightest nuclei. The symbol M3YE stands here for the version of the M3Y obtained in Ref. [23] by fitting the oscillator matrix elements derived from the $N N$ phase shifts by Elliott et al. [25]. The overlaps $r I_{01 / 2}^{\mathrm{IE}}(r)$ are also calculated for other $N N$ potentials, M3YR, M3YP, and M3YHJ constructed in Refs. [23,26] from the $G$-matrix elements of the Reid, Paris, and Hamada-Johnston potentials, and for the $N N$ potential GPT [27]. These overlaps, plotted in Fig. 1(b), were calculated with $r_{0}=1.51 \mathrm{fm}$, which reproduces the rms radius of the Hulthen wave function and gives the location of the maximum of the oscillator wave function $\varphi_{0 s}(r)$ at the same $r$ as the maximum of the realistic deuteron wave function obtained with the AV18 potential [28]. The overlap function $I_{01 / 2}^{\mathrm{IE}}(r)$ obtained with any of the M3Y potentials has a node at $r \sim$ $0.5 \mathrm{fm}$ because these potentials have a hard core whereas the soft-core GPT potential gives a nodeless behavior at $r \rightarrow 0$. The wave function at these distances is not important for many observables. The realistic deuteron wave function is best reproduced by the M3YE, M3YHJ, and GPT potentials. Table I shows the SFs, ANCs, and rms radii of $I_{01 / 2}^{\mathrm{IE}}(r)$ for all $\tilde{V}^{\text {eff }}$ used in the present calculations. The $s$-wave probability in the deuteron, $P_{s}=0.9424$, obtained with AV18 [28], is best reproduced by the M3YE, M3YHJ, and GPT potentials and the ANCs obtained with these potentials are close to the experimental value of $0.8781(44) \mathrm{fm}^{1 / 2}$ obtained in Ref. [29]. 

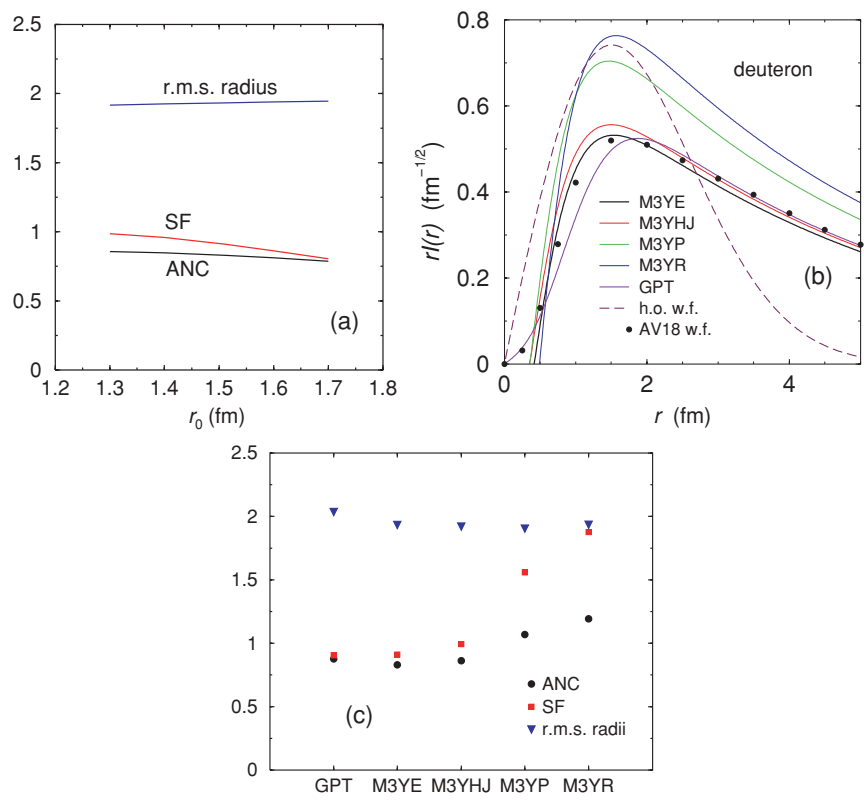

FIG. 1. (Color online) Spectroscopic factors, ANCs, and rms radii for $\langle d \mid p\rangle$ calculated with M3YE (a) as a function of oscillator radius $r_{0}$ and (c) as a function of the chosen effective $N N$ potential. (b) The overlap functions $r I_{01 / 2}^{\mathrm{IE}}(r)$ obtained with several effective $N N$ potentials in comparison to the harmonic oscillator (h.o.) wave function $\varphi_{0 s}(r)$ and the realistic deuteron wave function obtained with the AV18 potential in [28].

The rms radus of $I_{01 / 2}^{\mathrm{IE}}(r)$ obtained with $\mathrm{M} 3 \mathrm{YE}$ is very close to the experimental value of 1.953(3) fm from Ref. [28].

Although all M3Y potentials have the same long-range part determined by the one-pion-exchange potential, they strongly differs at small distances. The M3YR potential has the strongest short-range Yukawa terms, the first of which is twice as strong as in M3YE and the second is about $76 \%$ stronger. The resulting zeroth and second moments of this potential in the triplet-even channel are the largest [23]. The sum of the two short-range Yukawa terms give the major contribution to the overlap integral for all $r$. The M3YR has the strongest short-range part and, as the result, gives the largest (and unphysical) normalization of the deuteron wave function and on this basis should be discarded. The same conclusion is applicable to M3YP.

TABLE I. The spectroscopic factors, ANCs (in $\mathrm{fm}^{1 / 2}$ ), and rms radii (in fm) of the overlap function $I_{01 / 2}^{\mathrm{IE}}(r)$ calculated for four M3Y potentials and for GPT in comparison to those obtained from the realistic potential AV18 and to the experimental values.

\begin{tabular}{llll}
\hline \hline$\tilde{V}^{\text {eff }}$ & SF & \multicolumn{1}{c}{ ANC } & $\left\langle r^{2}\right\rangle^{1 / 2}$ \\
\hline M3YE & 0.91 & 0.830 & 1.933 \\
M3YHJ & 0.99 & 0.861 & 1.920 \\
M3YP & 1.56 & 1.068 & 1.904 \\
M3YR & 1.88 & 1.193 & 1.933 \\
GPT & 0.91 & 0.877 & 2.035 \\
AV18 & 0.9424 & 0.885 & 1.967 \\
Exp. & & $0.8781(44)$ & $1.953(3)$ \\
\hline \hline
\end{tabular}

Thus, it is possible to construct an overlap $I_{01 / 2}^{\mathrm{IE}}(r)$ that is reasonably close to the realistic deuteron wave function from an oscillator wave function that represents the lowest possible shell-model space. It is interesting that a very reasonable result is obtained with the effective M3YE potential constructed from the experimental oscillator matrix elements. This cannot be accidental. Indeed, $r I_{01 / 2}^{\mathrm{IE}}(r)$ can be presented as $\sum_{n}\left\langle G_{0}\left(r, r^{\prime}\right) \mid \varphi_{n 0}\left(r^{\prime}\right)\right\rangle V_{n 0}^{00}$, where $V_{n^{\prime} l^{\prime}}^{n l}=\left\langle n^{\prime} l^{\prime}\left\|\tilde{V}^{\text {eff }}\right\| n l\right\rangle$ are oscillator matrix elements of the $N N$ interaction. M3YE has been constructed to reproduce $V_{n^{\prime} l^{\prime}}^{n l}$ restored from the $N N$ scattering phase shifts. The latter can be similarly represented by $\sum_{n}\left\langle j_{0}(k r) \mid \varphi_{n 0}(r)\right\rangle V_{n 0}^{00}$, where $j_{0}$ is the spherical Bessel function. It contains the same matrix elements $V_{n 0}^{00}$ as the deuteron bound-state wave function.

\section{B. $A=3$}

To calculate the overlap functions $\left\langle\left. d\right|^{3} \mathrm{H}\right\rangle$ and $\left\langle\left. d\right|^{3} \mathrm{He}\right\rangle$ the model wave function $\Phi_{3}$ for the $A=3$ system has been taken as

$$
\begin{aligned}
\Phi_{3}\left(\boldsymbol{\xi}_{1}, \boldsymbol{\xi}_{2}\right)= & \frac{4}{r_{0}^{3} \sqrt{\pi}} \exp \left(-\frac{\xi_{1}^{2}+\xi_{2}^{2}}{2 r_{0}^{2}}\right) \\
& \times Y_{00}\left(\hat{\boldsymbol{\xi}}_{1}\right) Y_{00}\left(\hat{\boldsymbol{\xi}}_{2}\right) \chi^{S T}(1,2,3) .
\end{aligned}
$$

Here, $\xi_{1}$ and $\xi_{2}$ are the normalized Jacobi coordinates [30] and $\chi^{S T}(1,2,3)$ is the antisymmetric spin-isospin wave function corresponding to spin $S=1 / 2$ and isospin $T=1 / 2$. In Fig. 2(a), the SFs, ANCs, and the rms radii are shown as a function of $r_{0}$ chosen in the same range as for $\langle d \mid \mathrm{p}\rangle$. But, unlike in the $\langle d \mid \mathrm{p}\rangle$ case, in this range the SFs are equally sensitive to $r_{0}$ and to the choice of $N N$ potential. The overlap functions calculated with different effective potentials $\tilde{V}^{\text {eff }}$ are shown in Fig. 2(b) and the corresponding SFs and ANCs are given in Table II. In these calculations, the oscillator parameter $r_{0}=1.53 \mathrm{fm}$, chosen to provide the correct rms radius of triton, is close to the $r_{0}=1.51 \mathrm{fm}$ used for the deuteron in the previous section. As in the case of the deuteron, the M3YR and M3YP potentials give an SF that is larger than the upper
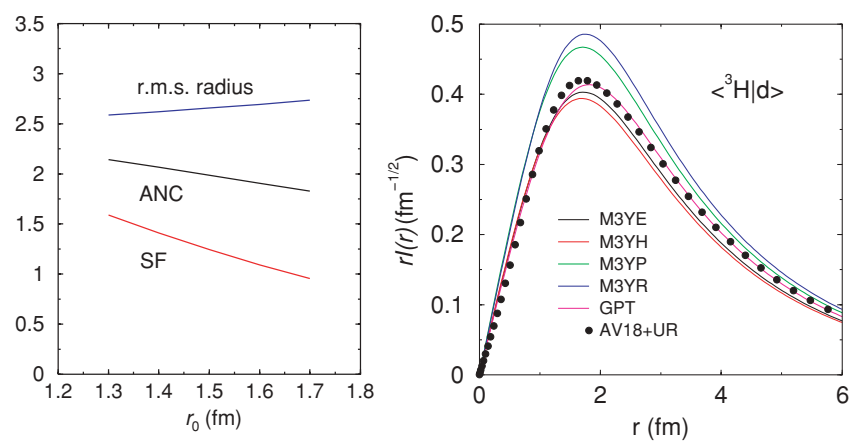

FIG. 2. (Color online) (a) Spectroscopic factors, ANCs, and rms radii for $\left\langle\left. d\right|^{3} \mathrm{H}\right\rangle$ calculated with M3YE as a function of oscillator radius $r_{0}$ chosen to be the same both for the deuteron and the triton wave functions. (b) The overlap functions $r I_{01 / 2}^{\mathrm{IE}}(r)$ calculated for several effective $N N$ potentials in comparison to those obtained from the three-body calculations with AV18 + UR in Ref. [31]. The choice of $r_{0}$ is discussed in the text. 
TABLE II. The SFs, ANCs (in $\mathrm{fm}^{-1 / 2}$ ), and $\mathrm{rms}$ radii (in $\mathrm{fm}$ ) for the overlaps $\left\langle\left. d\right|^{3} \mathrm{H}\right\rangle$ and $\left\langle\left. d\right|^{3} \mathrm{He}\right\rangle$ calculated for four M3Y potentials and for GPT in comparison to those obtained from ab initio three-body calculations with AV18 + UR [31]. Experimental ANC values are also shown. The experimental ${ }^{3} \mathrm{He}$ ANC has been derived from the ${ }^{3} \mathrm{H}$ ANC using the ratio $C_{3^{\mathrm{He}}}^{2} / C_{t}^{2}$ obtained from $a b$ initio calculations.

\begin{tabular}{|c|c|c|c|c|c|c|}
\hline \multirow[t]{2}{*}{$\tilde{V}^{\text {eff }}$} & \multicolumn{3}{|c|}{${ }^{3} \mathrm{H}$} & \multicolumn{3}{|c|}{${ }^{3} \mathrm{He}$} \\
\hline & SF & ANC & $\left\langle r^{2}\right\rangle^{1 / 2}$ & $\mathrm{SF}$ & ANC & $\left\langle r^{2}\right\rangle^{1 / 2}$ \\
\hline M3YE & 1.20 & 1.97 & 2.668 & 1.21 & 1.96 & 2.724 \\
\hline M3YHJ & 1.15 & 1.90 & 2.649 & 1.16 & 1.90 & 2.701 \\
\hline M3YP & 1.61 & 2.26 & 2.656 & 1.62 & 2.25 & 2.712 \\
\hline M3YR & 1.74 & 2.38 & 2.687 & 1.75 & 2.36 & 2.743 \\
\hline GPT & 1.30 & 2.12 & 2.737 & 1.31 & 2.12 & 2.794 \\
\hline $\mathrm{AV}+\mathrm{UR}$ & 1.30 & 2.12 & & 1.32 & 2.13 & \\
\hline Exp. & & $2.07(2)$ & & & $2.08(2)$ & \\
\hline
\end{tabular}

limit of $3 / 2$ imposed by the sum rule that follows from the normalization of the three-body wave function to unity. Unlike in the deuteron, where only the triplet-even potential is needed, the $A=3$ overlaps have contributions from the singlet-even partial waves as well. The short-range of M3YR and M3YP in these partial waves is stronger than in M3YE and M3YHJ, although to a lesser extent than for triplet partial waves, which causes the unrealistic normalization of the overlap.

For three potentials, M3YE, M3YHJ, and GPT, the SFs obtained clearly show the reduction from the $S_{l j}^{\mathrm{DE}}$ value of 1.5. Their values, $1.15,1.2$ and 1.3 , are close to the SF of the $a b$ initio calculations, $S_{a b}=1.3$, obtained as the norm of the $\left\langle\left. d\right|^{3} \mathrm{H}\right\rangle$ overlap calculated in Ref. [31] using the expansion of $\Psi_{3}$ onto hyperspherical harmonics basis functions [32]. The $a b$ initio overlap $I_{a b}(r)$ is shown in Fig. 2(b). The overlap $I_{01 / 2}^{\mathrm{IE}}(r)$ has a shape similar to that of $I_{a b}(r)$.

One important property of the $\left\langle\left. d\right|^{3} \mathrm{H}\right\rangle$ or the $\left\langle\left. d\right|^{3} \mathrm{He}\right\rangle$ overlap is their ANC, $C_{t}$ or $C^{{ }^{3} \mathrm{He}}$. Over the past two decades these ANCs have been used to extract the ANCs $C_{A}$ for a range of light nuclei using the peripheral transfer $(d, t)$ and $\left({ }^{3} \mathrm{He}, d\right)$ reactions. Since the amplitudes of these reactions contain the products $C_{t}^{2} C_{A}^{2}$ or $C_{{ }^{3} \mathrm{He}}^{2} C_{A}^{2}$ the accuracy of $C_{A}$ obtained depends on the accuracy of $C_{t}$ and $C_{3} \mathrm{He}$. The experimental value $C_{t}=2.07 \pm 0.02 \mathrm{fm}^{-1 / 2}$, shown in Table II and used to analyze the $(d, t)$ reactions, has been obtained from the vertex constant $G_{t}^{2}=1.34 \pm 0.02 \mathrm{fm}$ derived in Ref. [33] from the analysis of the high-precision data on the $d(d, p) t$ reaction at different incident energies. The method of analysis is explained in Ref. [29]. However, other values for $G_{t}^{2}$, ranging from 0.92 to $1.5 \mathrm{fm}$, are reviewed in Ref. [20]. Why these values are not used in the analysis of the $(d, t)$ reactions remains to be clarified. No experimental determinations are available for the mirror ANC $C_{3} \mathrm{He}$. To analyze the $\left({ }^{3} \mathrm{He}, d\right)$ reactions, two different values for $C_{3} \mathrm{He}$ have been used. The first value was assumed to be equal to $C_{t}, C^{{ }^{3} \mathrm{He}}=2.07 \pm 0.02 \mathrm{fm}^{-1 / 2}$, and has been used in the ANC compilation produced by Artemov et al. [34]. The second value, $C_{{ }^{3} \mathrm{He}}=1.97 \pm 0.03 \mathrm{fm}^{-1 / 2}$, was derived in Ref. [35] from the difference between the triton and ${ }^{3}$ He vertex constants deduced from an analysis of $s$-wave $n-d$ and $p$-d scattering. This value has been used in a number of $\left({ }^{3} \mathrm{He}, d\right)$ studies performed mainly at Texas A\&M University. However, Artemov et al. [36] pointed out that this second value gives the ratio $\mathcal{R}=C_{{ }^{3} \mathrm{He}}^{2} / C_{t}^{2}=0.91$, which contradicts relations imposed by mirror symmetry of the $N N$ interactions. On the basis of a potential model, in which the nuclear $n-d$ and $p-d$ potentials are the same, they deduced that $\mathcal{R}$ should be equal to 1.015 . A very close value, $\mathcal{R}=1.000$, is obtained if the analytical formula from Ref. [37] is used for $\mathcal{R}$. The $a b$ initio calculations predict that $\mathcal{R}=1.009$. The ratio $\mathcal{R}$ obtained in the inhomogeneous-equation approach of the present work ranges from 1.000 to 1.01 . Thus, all $C_{A}^{2}$ values obtained using the $C_{{ }^{3} \mathrm{He}}^{2}$ from Ref. [35] are overestimated by $\sim 10 \%$. It should be noted, however, that the experimental $C_{t}$ value is $12 \%$ smaller than the $a b$ initio predictions. This may indicate that either the experimental determination of $C_{t}$ or the $a b$ initio calculations or both should be reconsidered. In particular, a slightly larger $a b$ initio value for the ANC can result from using the AV18 potential, which slightly overestimates the deuteron ANC. The present calculations with GPT support the $a b$ initio value whereas the M3YE and M3YHJ potentials give a value that is smaller than $C_{t}$ from Ref. [33] but is still within the range that corresponds to the nuclear vertex constants from Ref. [20]. The ANCs obtained with the other two potentials, M3YR and M3YP, are clearly too large.

\section{C. $A=4$}

The overlap functions $\left\langle{ }^{4} \mathrm{He} \mid{ }^{3} \mathrm{H}\right\rangle$ and $\left\langle{ }^{4} \mathrm{He} \mid{ }^{3} \mathrm{He}\right\rangle$ have been calculated using the model wave function $\Phi_{3}$ for ${ }^{3} \mathrm{H}$ and ${ }^{3} \mathrm{He}$ given by Eq. (18) and the model wave function $\Phi_{4}$ for ${ }^{4} \mathrm{He}$ taken as

$$
\begin{aligned}
\Phi_{4}\left(\boldsymbol{\xi}_{1}, \boldsymbol{\xi}_{2}, \boldsymbol{\xi}_{3}\right)= & \frac{8}{r_{0}^{9 / 2} \pi^{3 / 4}} \exp \left(-\frac{\xi_{1}^{2}+\xi_{2}^{2}+\xi_{3}^{2}}{2 r_{0}^{2}}\right) Y_{00}\left(\hat{\boldsymbol{\xi}}_{1}\right) \\
& \times Y_{00}\left(\hat{\boldsymbol{\xi}}_{2}\right) Y_{00}\left(\hat{\boldsymbol{\xi}}_{3}\right) \chi^{S T}(1,2,3,4),
\end{aligned}
$$

where $\boldsymbol{\xi}_{1}, \boldsymbol{\xi}_{2}$, and $\boldsymbol{\xi}_{3}$ are the normalized Jacobi coordinates and $\chi^{S T}(1,2,3,4)$ is the antisymmetric spin-isospin wave function corresponding to spin $S=0$ and isospin $T=0$. The oscillator parameter $r_{0}\left({ }^{4} \mathrm{He}\right)=1.33 \mathrm{fm}$, which reproduces the radius of ${ }^{4} \mathrm{He}$, differs strongly from $r_{0}\left({ }^{3} \mathrm{H}\right)$. For the lightest nuclei, the calculations with $r_{0}\left({ }^{4} \mathrm{He}\right) \neq r_{0}\left({ }^{3} \mathrm{H}\right)$ are easy to carry out. Table III shows how different choices of $r_{0}\left({ }^{4} \mathrm{He}\right)$ and $r_{0}\left({ }^{3} \mathrm{H}\right)$ influence the SFs, ANCs, and rms radii of $\left\langle{ }^{4} \mathrm{He} \mid{ }^{3} \mathrm{He}\right\rangle$ at a fixed effective $N N$ potential $\tilde{V}^{\text {eff }}$. The SF obtained with $r_{0}\left({ }^{4} \mathrm{He}\right)=$ $1.33 \mathrm{fm}$ and $r_{0}\left({ }^{3} \mathrm{He}\right)=1.53 \mathrm{fm}$, chosen to reproduce the rms radii of ${ }^{4} \mathrm{He}$ and ${ }^{3} \mathrm{He}$, is close to the one obtained with an averaged value of $r_{0}^{\text {av }}=\left[r_{0}\left({ }^{4} \mathrm{He}\right)+r_{0}\left({ }^{3} \mathrm{He}\right)\right] / 2$ for both ${ }^{4} \mathrm{He}$ and ${ }^{3} \mathrm{He}$. However, the rms radius obtained with $r_{0}^{\text {av }}$ is larger than the one calculated with two different values of $r_{0}\left({ }^{4} \mathrm{He}\right)$ and $r_{0}\left({ }^{3} \mathrm{He}\right)$ and the difference between the corresponding ANCs is $4 \%$. It should be noted that the difference in $r_{0}$ for two neighboring nuclei is usually smaller than in the case of $\left\langle{ }^{4} \mathrm{He} \mid{ }^{3} \mathrm{He}\right\rangle$. In Table III, two cases are shown where the difference between $r_{0}\left({ }^{4} \mathrm{He}\right)$ and $r_{0}\left({ }^{3} \mathrm{He}\right)$ is typical for $0 p$-shell nuclei. For these cases, the difference in $r_{0}$ for ${ }^{4} \mathrm{He}$ and ${ }^{3} \mathrm{He}$ influences the ANC and the rms radius by less than $1 \%$, whereas the influence on the SFs is somewhat larger, between $1.5 \%$ and $3.0 \%$. 
TABLE III. The spectroscopic factors, ANCs (in $\mathrm{fm}^{-1 / 2}$ ), and $\mathrm{rms}$ radii $\left\langle r^{2}\right\rangle^{1 / 2}$ (in fm) of the overlap function $\left\langle\left.{ }^{4} \mathrm{He}\right|^{3} \mathrm{He}\right\rangle$ calculated for M3YE with different choices of oscillator parameter for ${ }^{3} \mathrm{He}$ and ${ }^{4} \mathrm{He}$.

\begin{tabular}{lcccc}
\hline \hline$r_{0}\left({ }^{3} \mathrm{He}\right)$ & $r_{0}\left({ }^{4} \mathrm{He}\right)$ & $\mathrm{SF}$ & ANC & $\left\langle r^{2}\right\rangle^{1 / 2}$ \\
\hline 1.53 & 1.33 & 1.292 & 5.282 & 1.903 \\
1.33 & 1.33 & 1.467 & 5.547 & 1.897 \\
1.53 & 1.53 & 1.092 & 5.441 & 1.979 \\
1.43 & 1.43 & 1.271 & 5.497 & 1.937 \\
1.46 & 1.40 & 1.292 & 5.467 & 1.934 \\
1.40 & 1.46 & 1.238 & 5.495 & 1.946 \\
\hline \hline
\end{tabular}

The $\left\langle{ }^{4} \mathrm{He} \mid{ }^{3} \mathrm{H}\right\rangle$ overlaps calculated for different choices of $\tilde{V}^{\text {eff }}$ are shown in Fig. 3 and their SFs, ANCs, and rms radii are given in Table IV. They are compared to the predictions of the ab initio calculations performed in a hyperspherical functions expansion method with the AV18 + IX interaction [32,38]. Similar to the $\langle d \mid p\rangle$ and $\left\langle\left. d\right|^{3} \mathrm{H}\right\rangle$ case, the M3YR and M3YP potentials give SFs that are significantly larger than those obtained in $a b$ initio calculations. Three other potentials give smaller SFs than the ab initio ones. The rms radii for most $N N$ effective potentials are smaller than those obtained in $a b$ initio calculations (see Table IV).

The present calculations with the same $N N$ potential $\tilde{V}^{\text {eff }}$ that gives reasonable results both for the deuteron and for ${ }^{3} \mathrm{H}$ and ${ }^{3} \mathrm{He}$ predicts that $S_{l j}^{\mathrm{IE}}$ should be reduced with respect to $S_{l j}^{\mathrm{DE}}$. The reduction factor is $0.645-0.665$ for M3YE, M3HJ, and GPT. The ab initio SF is also only $75 \%$ of the independentparticle-model value of 2 . Experimentally, the ${ }^{4} \mathrm{He}\left(e, e^{\prime} p\right)^{3} \mathrm{H}$ cross sections are reduced with respect to the independentparticle model by a factor of $0.7-0.8$ [1].

The ANCs from the present calculations deviate from the $a b$ initio values to the same extent as the SFs do since both are determined by the same source term. Experimental information on ${ }^{4} \mathrm{He}$ ANCs is not accurate. Several values for the ${ }^{4} \mathrm{He}$ vertex constant, obtained using various methods, quoted in the review by Blokhintsev [20], correspond to the ANCs ranging from 5.34 to $8.94 \mathrm{fm}^{-1 / 2}$. The $a b$ initio ANCs lie within the experimental range but the M3YE and M3YHJ

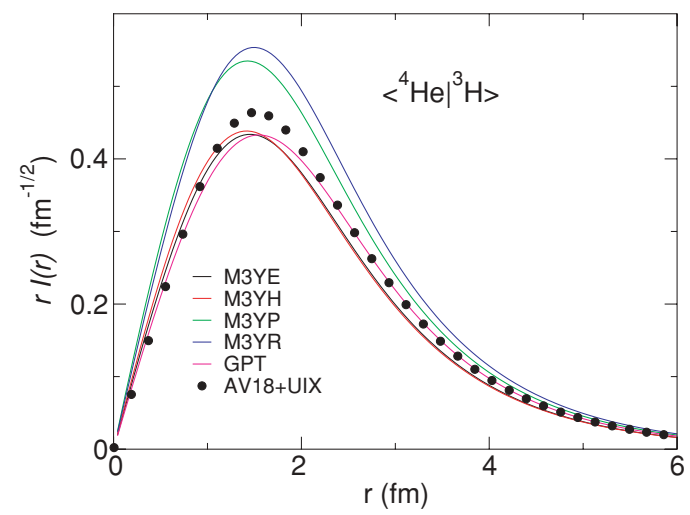

FIG. 3. (Color online) The overlap function $\left\langle{ }^{4} \mathrm{He} \mid{ }^{3} \mathrm{H}\right\rangle$ calculated for several effective $N N$ potentials in comparison to the one obtained from the four-body calculations with the AV18 + IX potential in Ref. [38].
TABLE IV. The SFs, ANCs (in $\mathrm{fm}^{-1 / 2}$ ), and rms radii (in fm) for the overlap functions $\left\langle{ }^{4} \mathrm{He} \mid{ }^{3} \mathrm{H}\right\rangle$ and $\left\langle{ }^{4} \mathrm{He} \mid{ }^{3} \mathrm{He}\right\rangle$ calculated for four M3Y potentials and for GPT in comparison to those derived from $a b$ initio calculations with the AV18 + IX potential [38].

\begin{tabular}{lccccccc}
\hline \hline$\tilde{V}^{\text {eff }}$ & \multicolumn{3}{c}{${ }^{3} \mathrm{H}$} & & \multicolumn{3}{c}{${ }^{3} \mathrm{He}$} \\
\cline { 2 - 4 } \cline { 6 - 8 } & SF & ANC & $\left\langle r^{2}\right\rangle^{1 / 2}$ & & SF & ANC & $\left\langle r^{2}\right\rangle^{1 / 2}$ \\
\hline M3YE & 1.29 & 5.35 & 1.910 & & 1.29 & 5.28 & 1.903 \\
M3YHJ & 1.31 & 5.27 & 1.884 & & 1.31 & 5.20 & 1.877 \\
M3YP & 1.96 & 6.42 & 1.889 & & 1.95 & 6.34 & 1.881 \\
M3YR & 2.11 & 6.97 & 1.939 & & 2.10 & 6.89 & 1.931 \\
GPT & 1.33 & 5.95 & 1.991 & & 1.34 & 5.87 & 1.981 \\
AV18 + IX & 1.52 & 6.18 & 1.970 & 1.50 & 6.02 & 1.965 \\
\hline \hline
\end{tabular}

potentials gives smaller ANCs. It is interesting to note that the ratio $\mathcal{R}=\left(C_{p} / C_{n}\right)^{2}$ is 1.03 , where $C_{p}$ and $C_{n}$ are the mirror proton and neutron ANC in ${ }^{4} \mathrm{He}$, respectively, obtained in the present calculations is in a good agreement with the ab initio value of 1.05 . Both agree well with the prediction $\mathcal{R}=1.04$ of the analytical formula from Ref. [37].

\section{D. $A=16$}

To calculate the overlaps $\left\langle{ }^{15} \mathrm{~N} \mid{ }^{16} \mathrm{O}\right\rangle$ and $\left\langle{ }^{15} \mathrm{O} \mid{ }^{16} \mathrm{O}\right\rangle$, the $A=15$ and $A=16$ wave functions were represented only by one $0 \hbar \omega$ shell-model configuration, which is equivalent to using a single Slater determinant, with only $0 s$ and $0 p$ shell-model orbitals occupied, divided by the wave function of the $0 s$ center-of-mass motion. The technical aspects of these calculations are discussed in the Appendix.

The oscillator radius $r_{0}$ was assumed to be the same both in ${ }^{15,16} \mathrm{O}$ and ${ }^{15} \mathrm{~N}$. The SFs, ANCs, and rms radii for $\left\langle{ }^{15} \mathrm{~N}\left(1 / 2^{-}\right) \mid{ }^{16} \mathrm{O}\right\rangle$ and $\left\langle{ }^{15} \mathrm{~N}\left(3 / 2^{-}\right) \mid{ }^{16} \mathrm{O}\right\rangle$, calculated with M3YE as a function of $r_{0}$, are shown in Figs. 4(a) and 4(b). The rms radius of $\left\langle{ }^{15} \mathrm{~N} \mid{ }^{16} \mathrm{O}\right\rangle$ has been determined from the $\left(e, e^{\prime} p\right)$ knockout. It is equal to 2.943(30) and 2.719(24) fm for ${ }^{15} \mathrm{~N}\left(1 / 2^{-}\right)$and ${ }^{15} \mathrm{~N}\left(3 / 2^{-}\right)$, respectively. To reproduce these values, $r_{0}$ should be approximately equal to $1.79 \mathrm{fm}$, which is close to $r_{0}^{\text {av }}=1.80 \mathrm{fm}$ averaged over the oscillator parameters of ${ }^{15} \mathrm{~N}$ and ${ }^{16} \mathrm{O}$ derived from electron scattering data [39]. With $r_{0}=1.79 \mathrm{fm}$ the spectroscopic factors $S_{l j}^{\mathrm{IE}}$, equal to 1.58 and 2.62 for ${ }^{15} \mathrm{~N}\left(1 / 2^{-}\right)$and ${ }^{15} \mathrm{~N}\left(3 / 2^{-}\right)$, respectively, are reduced from their $S_{l j}^{\mathrm{DE}}$ values of 2.133 and 4.267. The overlaps $r I_{l j}^{\mathrm{IE}}(r)$ calculated with different effective potentials $\tilde{V}^{\mathrm{eff}}$ and with $r_{0}^{a v}=1.80 \mathrm{fm}$ are shown in Figs. 4(c) and 4(d) where they are compared to the overlap functions deduced from ${ }^{16} \mathrm{O}\left(e, e^{\prime} p\right){ }^{15} \mathrm{~N}$ in Ref. [40]. All $r I_{l j}^{\mathrm{IE}}(r)$ have similar shapes but different normalizations.

The ratio $S^{\mathrm{IE}} / S^{\mathrm{DE}}$, shown in Fig. 5 for various potentials, follows the same trend observed in $A=3$ and $A=4$ nuclei in previous sections. According to Fig. 5, those potentials that give a reasonable normalization for $\langle d \mid p\rangle$ lead to the SF reduction in ${ }^{16} \mathrm{O}$. The best agreement with the $\operatorname{SF}$ from $\left(e, e^{\prime} p\right)$ is obtained only with M3YE, which gives the largest reduction of spectroscopic strength. The SFs, ANCs squared, and rms radii for this potential are presented in Table $\mathrm{V}$ in comparison to 

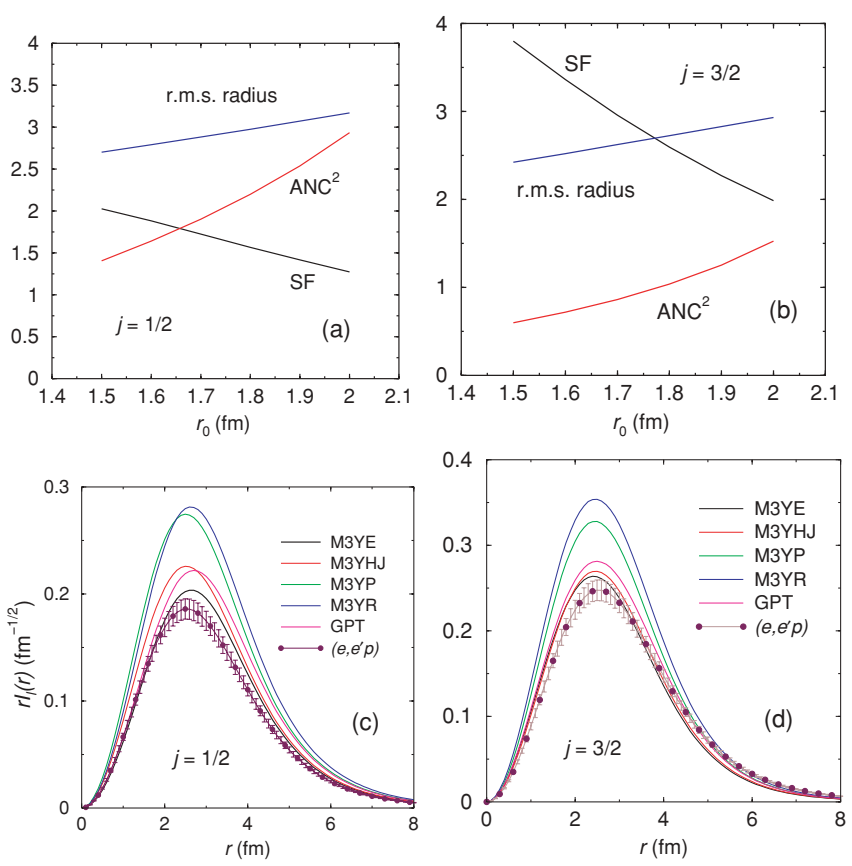

FIG. 4. (Color online) The SFs, squared ANCs, and rms radii for (a) $\left\langle{ }^{15} \mathrm{~N}\left(1 / 2^{-}\right) \mid{ }^{16} \mathrm{O}\right\rangle$ and (b) $\left\langle{ }^{15} \mathrm{~N}\left(3 / 2^{-}\right) \mid{ }^{16} \mathrm{O}\right\rangle$ as a function of $r_{0}$ calculated with the M3YE potential. The overlap functions $r I^{\mathrm{IE}}(r)$ for (c) $\left\langle{ }^{15} \mathrm{~N}\left(1 / 2^{-}\right) \mid{ }^{16} \mathrm{O}\right\rangle$ and (d) $\left\langle{ }^{15} \mathrm{~N}\left(3 / 2^{-}\right) \mid{ }^{16} \mathrm{O}\right\rangle$ calculated with $r_{0}=1.8 \mathrm{fm}$ for four M3Y potentials and for GPT in comparison with the overlap functions derived from the $\left(e, e^{\prime} p\right)$ reaction [2].

those derived from various experimental data. The rms radii are close to the experimental values whereas the SFs are slightly larger. As for ANCs, two different values are available from the analysis of the $\left({ }^{3} \mathrm{He}, d\right)$ transfer reactions in the literature, $C_{\text {exp }}^{2}=298(63) \mathrm{fm}^{-1}$ [34] and $C_{\exp }^{2}=192(26) \mathrm{fm}^{-1}$ [41]. The latter has been derived using a ${ }^{3} \mathrm{He}$ ANC that does not satisfy the mirror symmetry relations, as discussed in Sec. IV B. Using a corrected value for $C_{3} \mathrm{He}$ according to Ref. [36], one gets for ${ }^{16} \mathrm{O} C_{\exp }^{2}=175(29) \mathrm{fm}^{-1}$, which is much smaller than the value from Ref. [34]. The present calculation with M3YE gives $C^{2}=220 \mathrm{fm}^{-1}$, which is between these two experimental values.

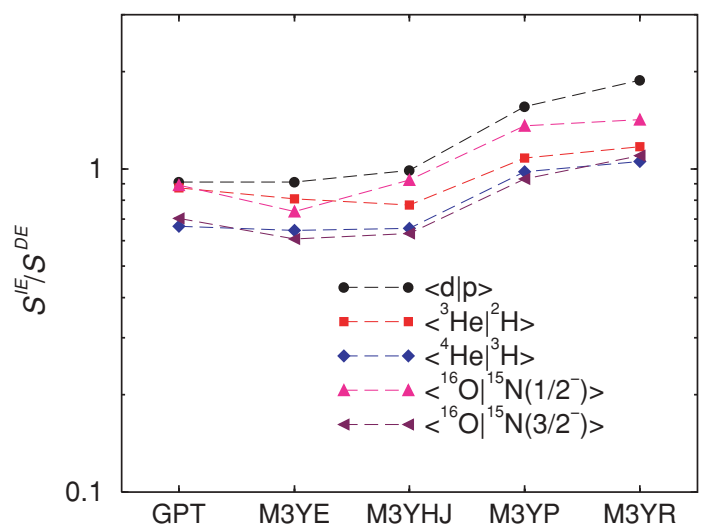

FIG. 5. (Color online) The ratio $S^{\mathrm{IE}} / S^{\mathrm{DE}}$ calculated for four M3YE potentials and for GPT for several overlap integrals.

\section{SHELL-MODEL CALCULATIONS FOR NUCLEI WITH CONFIGURATION MIXING}

The wave functions for $A>4$ nuclei cannot be described by a single shell-model configuration. They have a mixture of configurations with different total orbital momentum $L$ and spin $S$, which can be classified according to different irreducible representations of the permutation and the SU(4) groups. In the present work, the nuclear wave functions for $4<A<16$ have been chosen in the $0 \hbar \omega$ translationally invariant shell model in the supermultiplet scheme:

$$
\begin{aligned}
\Phi_{A}^{J_{A} M_{A}}= & \sum_{\gamma M_{L_{A}} M_{S_{A}} M_{T_{A}}}\left(L_{A} M_{L_{A}} S_{A} M_{S_{A}} \mid J_{A} M_{A}\right) \\
& \times \alpha_{\gamma}\left|A N \gamma M_{L_{A}} M_{S_{A}} M_{T_{A}}\right\rangle,
\end{aligned}
$$

where $N$ is the total number of oscillator quanta in nucleus $A, \gamma=\{[f](\lambda \mu) \beta L S T\}, T$ is the total isospin, $[f]$ is the Young diagram determining the permutational symmetry of the orbital part of the wave function, $(\lambda \mu)$ is the Elliott symbol defining the SU(3) symmetry of the state, and $\beta$ serves to distinguish any degenerate states. $\left(L_{A} M_{L_{A}} S_{A} M_{S_{A}} \mid J_{A} M_{A}\right)$ is the Clebsch-Gordan coefficient and $\alpha_{\gamma}$ is the weight of the configuration $\gamma$ found from the diagonalization of the shellmodel Hamiltonian in the $0 \hbar \omega$ space with some effective $N N$ interaction. The details of the calculation of the source term using the wave functions (20) are presented in the Appendix.

In earlier calculations [18,19,21,24], the coefficients $\alpha_{\gamma}$ were taken from the work of Boyarkina [42], where they were found by diagonalizing the shell-model Hamiltonian with the Rosenfeld interaction [43]. In the present work, these results are compared to those obtained with the more popular CohenKurath interaction CK816 [44] and with the recent Millener interaction [45], which provides the best fit to the spectra of the $0 p$-shell nuclei. In these calculations, M3YE was used as the effective interaction $\tilde{V}^{\text {eff }}$. No renormalization of the M3YE potential, similar to the one in the previous publication [11], has been done. The oscillator parameter was assumed to be the same in both $A$ and $A-1$ and equal to $r_{0}^{\text {av }}=\left(r_{0}^{A-1}+\right.$ $\left.r_{0}^{A}\right) / 2$, where $r_{0}^{A-1}$ and $r_{0}^{A}$ were taken from analyses of electron scattering in Ref. [39].

The results of the calculations are presented in two tables. Table VI contains the ANCs calculated with three shell-model interactions for those overlaps where experimental values $C_{\exp }$ are available. Table VII displays the ANCs, rms radii, and SFs $S^{\mathrm{IE}}$ and $S^{\mathrm{DE}}$ calculated with the Millener interaction. For lighter nuclei, where the $a b$ initio VMC calculations are available, the corresponding VMC SFs are also presented. Also shown are the experimental SFs obtained from the $\left(e, e^{\prime} p\right)$, proton knockout, and those $(d, p)$ and $(p, d)$ reactions where adiabatic theory, in combination with the Hartree-Fock geometry for the transferred neutron potential, was used to analyze the experimental data.

\section{A. $A=15$}

The overlap $\left\langle{ }^{15} \mathrm{O} \mid{ }^{14} \mathrm{~N}\right\rangle$ is of interest in connection with the slowest $p p$ chain reaction ${ }^{14} \mathrm{~N}(p, \gamma){ }^{15} \mathrm{O}$ that determines the age of the Galaxy and thus helps to establish limits on the age 
TABLE V. The spectroscopic factors $S^{\mathrm{DE}}$ and $S^{\mathrm{IE}}$, squared ANCs, $C_{l j}^{2}$ (in fm ${ }^{-1}$ ), and $\mathrm{rms}$ radii $\left\langle r^{2}\right\rangle^{1 / 2}$ (in fm) for the $\left\langle{ }^{15} \mathrm{O} \mid{ }^{16} \mathrm{O}\right\rangle$ and $\left\langle{ }^{15} \mathrm{~N} \mid{ }^{16} \mathrm{O}\right\rangle$ overlaps calculated with the M3YE potential in comparison to those derived experimentally.

\begin{tabular}{|c|c|c|c|c|c|c|c|c|}
\hline \multirow[t]{2}{*}{$J^{\pi}$} & \multirow[t]{2}{*}{$S_{l j}^{\mathrm{DE}}$} & \multirow[t]{2}{*}{$S_{l j}^{\mathrm{IE}}$} & \multicolumn{2}{|c|}{$S_{\text {exp }}$} & \multirow[t]{2}{*}{$C_{l j}^{2}$} & \multirow{2}{*}{$\begin{array}{c}C_{\mathrm{exp}}^{2} \\
\left({ }^{3} \mathrm{He}, d\right)\end{array}$} & \multirow[t]{2}{*}{$\left\langle r^{2}\right\rangle^{1 / 2}$} & \multirow[t]{2}{*}{$\left\langle r^{2}\right\rangle_{\exp }^{1 / 2}$} \\
\hline & & & $\left(e, e^{\prime} p\right)$ & $\overline{\text { knockout }}$ & & & & \\
\hline${ }^{15} \mathrm{~N}\left(1 / 2^{-}\right)$ & 2.13 & 1.57 & $1.27(13)^{\mathrm{a}}$ & $1.10(7)^{\mathrm{b}}$ & 220 & $298(63) ;^{c} 175(29)^{d}$ & 2.975 & $2.943(30)^{\mathrm{a}}$ \\
\hline${ }^{15} \mathrm{O}\left(1 / 2^{-}\right)$ & 2.13 & 1.48 & & $0.92(6)^{\mathrm{b}}$ & 140 & & 2.936 & \\
\hline${ }^{15} \mathrm{~N}\left(3 / 2^{-}\right)$ & 4.26 & 2.59 & $2.25(22)^{\mathrm{a}}$ & $2.24(13)^{\mathrm{b}}$ & 1038 & & 2.725 & $2.719(24)^{\mathrm{a}}$ \\
\hline${ }^{15} \mathrm{O}\left(3 / 2^{-}\right)$ & 4.26 & 2.52 & & $1.84(10)^{\mathrm{b}}$ & 644 & & 2.699 & \\
\hline
\end{tabular}

${ }^{a}$ Reference [40]

${ }^{\mathrm{b}}$ Obtained from high-energy knockout reactions as $S_{\mathrm{exp}}=R_{s} S_{\text {th }}$ using $R_{s}=\sigma_{\text {th }} / \sigma_{\text {exp }}$ and $S_{\text {th }}$ calculated in the $4 \hbar \omega$ model space in Ref. [4].

${ }^{\mathrm{c}}$ Reference [34].

${ }^{\mathrm{d}}$ This is a corrected value of the ANC from Ref. [41] based on the updated value of the ANC for ${ }^{3} \mathrm{He}$.

of the universe [46]. The ANCs that determine this capture rate have been studied using the peripheral transfer reaction $\left({ }^{3} \mathrm{He}, d\right)[34,47,48]$. The ANCs from Ref. [48], presented in Table VI, are corrected according to the updated $C_{{ }^{3} \mathrm{He}}^{2}$ value, as explained in Sec. IV B. It should be noticed that the $\left.{ }^{14} \mathrm{~N}\left({ }^{3} \mathrm{He}, d\right)\right)^{15} \mathrm{O}$ angular distributions do not distinguish between the contributions from $j=1 / 2$ and $j=3 / 2$. In Ref. [48], these contributions have been fixed according to the shell-model ratio $S_{p 3 / 2}^{\mathrm{DE}} / S_{p 1 / 2}^{\mathrm{DE}}$ of the corresponding $\mathrm{SFs}$, which does not guarantee the correct ratio $C_{p 3 / 2} / C_{p 1 / 2}$. It is not clear what was used for this ratio in Ref. [34] either, so the uncertainties of the ANCs from this work can be larger.

The choice of the shell-model interaction that determine the weights $\alpha_{\gamma}$ in ${ }^{14} \mathrm{~N}$ does not strongly influence the largest ANC in ${ }^{15} \mathrm{O}\left(\frac{1}{2}^{-}\right), C_{p 1 / 2}^{2}$. However, all other ANCs change strongly because of cancellations between the contributions from different configurations in ${ }^{14} \mathrm{~N}$. Interestingly, the cancellations between these components are also responsible for slowness of the ${ }^{14} \mathrm{C} \beta^{-}$decay, which is hindered by about six orders of magnitude with respect to an allowed decay [45]. The Millener interaction, fitted to satisfy the cancellation between the $\left|[4442]^{13} \mathrm{~S}\right\rangle$ and $\left|[4433]^{11} \mathrm{P}\right\rangle$ components in the amplitude of the ${ }^{14} \mathrm{C} \beta^{-}$decay, gives the best agreement between the theoretical and experimental ANCs for ${ }^{15} \mathrm{O}$.

The spectroscopic factor $S_{p 1 / 2}^{\mathrm{IE}}$ for ${ }^{15} \mathrm{O}\left(1 / 2^{-}\right)$, given in Table VII, is close to $S_{p 1 / 2}^{\mathrm{DE}}$ for all three shell-model interactions used, $R_{s}$ being $0.96-0.97$. For $S_{p 3 / 2}^{\mathrm{DE}}$, the $\mathrm{SF}$ reduction is much larger, $R_{s}=0.65-0.75$. In comparison to the experimental $\mathrm{SF}$, the reduction is not sufficient. Renormalization of $\tilde{V}^{\text {eff }}$ is required to achieve agreement with the experimental value. For the excited state ${ }^{15} \mathrm{O}\left(3 / 2^{-}\right)$, where the cancellations from different components in ${ }^{14} \mathrm{~N}$ are crucial, $R_{s}$ depends strongly on the shell-orbit occupancies in ${ }^{14} \mathrm{~N}$ and varies within the range of $1.38-1.73$ for $p_{1 / 2}$ and $0.6-2.63$ for $p_{3 / 2}$.

\section{B. $A=14$}

The ANCs for the $\left\langle{ }^{14} \mathrm{~N} \mid{ }^{13} \mathrm{C}\right\rangle$ overlap have been determined from the ${ }^{13} \mathrm{C}\left({ }^{3} \mathrm{He}, d\right){ }^{14} \mathrm{~N}[36,49]$ and ${ }^{13} \mathrm{C}\left({ }^{14} \mathrm{~N},{ }^{13} \mathrm{C}\right){ }^{14} \mathrm{~N}[50]$ reactions in connection with the low-energy radiative capture reaction ${ }^{13} \mathrm{C}(p, \gamma){ }^{14} \mathrm{~N}$. The ANCs obtained from two different $\left({ }^{3} \mathrm{He}, d\right)$ studies agree with each other whereas the heavy-ion transfer gives slightly different values. As in the case of $\left\langle{ }^{15} \mathrm{O} \mid{ }^{14} \mathrm{~N}\right\rangle$, the best agreement between the theoretical and experimental ANCs is obtained with the Millener interaction, apart from the $\left\langle{ }^{14} \mathrm{~N}\left(2^{+}\right) \mid{ }^{13} \mathrm{C}\right\rangle$. The latter depends only on the weights $\alpha_{\gamma}$ of the ${ }^{13} \mathrm{C}$ components as the wave function of ${ }^{14} \mathrm{~N}\left(2^{+}\right)$is described by only one shell-model configuration, $\left|[4442]{ }^{13} \mathrm{D}\right\rangle$. The small value of the ANC for $\left\langle{ }^{14} \mathrm{~N}\left(2^{+}\right) \mid{ }^{13} \mathrm{C}\right\rangle$ is the result of destructive interference from the large component $\left.\mid[4432]]^{24} \mathrm{D}\right\rangle$ in ${ }^{13} \mathrm{C}$. This can be an indication that further tuning of the ${ }^{13} \mathrm{C}$ wave function is needed.

The ANC of the mirror overlap $\left\langle{ }^{14} \mathrm{~N} \mid{ }^{13} \mathrm{~N}\right\rangle$ has been determined from the $(d, t)$ reaction in Ref. [51]. According to the analytical formula from Ref. [37], the ratio $\mathcal{R}=$ $\left(C_{p} / C_{n}\right)^{2}$ for mirror ANCs in ${ }^{14} \mathrm{~N}$ should be equal to 1.39 . However, the experimental ratio, $\mathcal{R}_{\exp }=1.12(30)$, calculated using the ANCs from the $\left({ }^{3} \mathrm{He}, d\right)$ and the $(d, t)$ reactions can tolerate only the highest $C_{p}^{2} \approx 19.2 \mathrm{fm}^{-1}$ and the lowest $C_{n}^{2} \approx 13.5 \mathrm{fm}^{-1}$ values from the intervals determined by their experimental error bars. The ANC obtained from the ${ }^{13} \mathrm{C}\left({ }^{14} \mathrm{~N},{ }^{13} \mathrm{C}\right){ }^{14} \mathrm{~N}$ reaction fits the mirror symmetry relation better but, most likely, the small $\mathcal{R}_{\exp }$ value is an indication that the neutron ANC in ${ }^{14} \mathrm{~N}$ obtained from the $(d, t)$ reaction is too large.

The ANC for another overlap that involves $A=14$ and $A=13$ nuclei, $\left\langle{ }^{14} \mathrm{O} \mid{ }^{13} \mathrm{~N}\right\rangle$, has been studied in Refs. [52,53] in connection with the ${ }^{13} \mathrm{~N}(p, \gamma){ }^{14} \mathrm{O}$ capture in explosive hydrogen burning. The theoretical ANCs obtained with all three shell-model interactions agree very well with the experimental ones.

All calculated SFs $S^{\mathrm{IE}}$ for $A=14$ are reduced with respect to $S^{\mathrm{DE}}$. However, for ${ }^{14} \mathrm{~N}\left(1_{2}^{+}\right)$this reduction applies only to the sum $S_{p 1 / 2}^{\mathrm{IE}}+S_{p 3 / 2}^{\mathrm{IE}}$. The split between $S_{p 1 / 2}^{\mathrm{IE}}$ and $S_{p 3 / 2}^{\mathrm{IE}}$ in this case is different than in $S_{p 1 / 2}^{\mathrm{DE}}$ and $S_{p 3 / 2}^{\mathrm{DE}}$. The reduction factor for $A=14$ varies between 0.7 and 0.94 . The reduced value of the $\left\langle{ }^{14} \mathrm{~N} \mid{ }^{13} \mathrm{~N}\right\rangle \mathrm{SF}$ is still slightly larger than $S_{\text {exp }}$. The SF of another overlap, $\left\langle{ }^{14} \mathrm{C} \mid{ }^{13} \mathrm{C}\right\rangle$, is significantly larger than

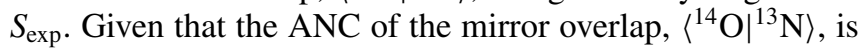
reasonably close to $C_{\exp }$, this means that either the shape of these overlaps is wrong or the experimental value for the ${ }^{14} \mathrm{C}$ $\mathrm{SF}$ is strongly underestimated. 


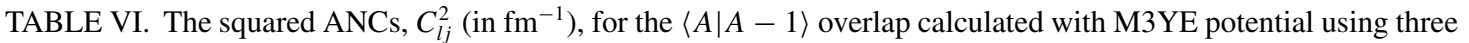
different sets of the shell-model wave functions in comparison to $C_{\mathrm{exp}}^{2}$ derived experimentally.

\begin{tabular}{|c|c|c|c|c|c|c|}
\hline \multirow[t]{2}{*}{$A$} & \multirow[t]{2}{*}{$A-1$} & \multirow[t]{2}{*}{$j$} & \multicolumn{3}{|c|}{$C_{l j}^{2}$} & \multirow[t]{2}{*}{$C_{\mathrm{exp}}^{2}$} \\
\hline & & & Boyarkina & CK816 & Millener & \\
\hline \multirow[t]{3}{*}{${ }^{15} \mathrm{O}\left(1 / 2^{-}\right)$} & ${ }^{14} \mathrm{~N}\left(1^{+}\right)$ & $1 / 2$ & 55.49 & 56.08 & 50.44 & $45.8(30)^{\mathrm{a}} ; 44.6(49)^{\mathrm{b}}$ \\
\hline & & $3 / 2$ & 0.117 & 0.561 & 2.07 & $\sim 1^{\mathrm{a}} ; 4.55(50)^{\mathrm{b}}$ \\
\hline & & sum & 55.61 & 56.64 & 52.51 & $46.8(30)^{\mathrm{a}} ; 49.1(54)^{\mathrm{b}} ; 63(14)^{\mathrm{c}}$ \\
\hline \multirow[t]{2}{*}{${ }^{15} \mathrm{O}\left(3 / 2^{-}\right)$} & ${ }^{14} \mathrm{~N}\left(1^{+}\right)$ & $1 / 2$ & 0.109 & 0.222 & 0.511 & $0.53(4)^{\mathrm{b}} ; 0.46(6)^{\mathrm{b}} ; 0.46(10)^{\mathrm{c}}$ \\
\hline & & $3 / 2$ & 0.186 & $10^{-3}$ & $5.7 \times 10^{-6}$ & \\
\hline \multirow[t]{2}{*}{${ }^{14} \mathrm{~N}\left(1_{1}^{+}\right)$} & ${ }^{13} \mathrm{C}\left(1 / 2^{-}\right)$ & $1 / 2$ & 23.02 & 19.13 & 18.49 & $16.7(25)^{\mathrm{d}} ; 15.9(12)^{\mathrm{e}} ; 18.6(12)^{\mathrm{f}}$ \\
\hline & & $3 / 2$ & 0.024 & 0.195 & 0.249 & $0.84(12)^{\mathrm{d}} ; 0.79(12)^{\mathrm{e}} ; 0.93(14)^{\mathrm{f}}$ \\
\hline${ }^{14} \mathrm{~N}\left(0^{+}\right)$ & ${ }^{13} \mathrm{C}\left(1 / 2^{-}\right)$ & $1 / 2$ & 14.8 & 15.2 & 13.6 & $12.1(18)^{\mathrm{d}} ; 14.3(10)^{\mathrm{e}} ; 8.9(9)^{\mathrm{f}}$ \\
\hline \multirow[t]{2}{*}{${ }^{14} \mathrm{~N}\left(1_{2}^{+}\right)$} & ${ }^{13} \mathrm{C}\left(1 / 2^{-}\right)$ & $1 / 2$ & 0.2747 & 0.616 & 1.525 & $2.15(31)^{\mathrm{d}}, \mathrm{g} ; 2.6(2)^{\mathrm{e}} ; 2.8(3)^{\mathrm{f}}$ \\
\hline & & $3 / 2$ & 0.8179 & 0.6825 & 0.596 & \\
\hline${ }^{14} \mathrm{~N}\left(2^{+}\right)$ & ${ }^{13} \mathrm{C}\left(1 / 2^{-}\right)$ & $3 / 2$ & 0.0084 & 0.114 & 0.071 & $0.26(4)^{\mathrm{d}}$ \\
\hline${ }^{14} \mathrm{~N}\left(1_{1}^{+}\right)$ & ${ }^{13} \mathrm{~N}\left(1 / 2^{-}\right)$ & sum & 15.94 & 13.27 & 12.87 & $15.4(19)^{\mathrm{h}}$ \\
\hline${ }^{14} \mathrm{O}\left(0^{+}\right)$ & ${ }^{13} \mathrm{~N}\left(1 / 2^{-}\right)$ & $1 / 2$ & 31.12 & 31.68 & 31.85 & $29.0(43)^{\mathrm{i}} ; 29.4(52)^{\mathrm{j}}$ \\
\hline${ }^{13} \mathrm{C}\left(1 / 2^{-}\right)$ & ${ }^{12} \mathrm{C}\left(0^{+}\right)$ & $1 / 2$ & 2.768 & 2.289 & 2.067 & $2.46(31)^{\mathrm{h}} ; 2.39(9)^{\mathrm{k}}$ \\
\hline${ }^{13} \mathrm{C}\left(1 / 2^{-}\right)$ & ${ }^{12} \mathrm{C}\left(2^{+}\right)$ & $3 / 2$ & 6.815 & 7.299 & 6.597 & $10.4(12)^{\mathrm{h}}$ \\
\hline${ }^{13} \mathrm{~N}\left(1 / 2^{-}\right)$ & ${ }^{12} \mathrm{C}\left(0^{+}\right)$ & $1 / 2$ & 3.491 & 2.903 & 2.625 & $3.26(25)^{\mathrm{a}}$ \\
\hline \multirow[t]{3}{*}{${ }^{13} \mathrm{O}\left(3 / 2^{-}\right)$} & ${ }^{12} \mathrm{~N}\left(1^{+}\right)$ & $1 / 2$ & 3.343 & 3.393 & 3.254 & \\
\hline & & $3 / 2$ & 0.220 & 0.1614 & 0.361 & \\
\hline & & sum & 3.560 & 3.554 & 3.615 & $2.53(30)^{\mathrm{g}}{ }^{1}$ \\
\hline \multirow[t]{3}{*}{${ }^{12} \mathrm{~B}\left(1^{+}\right)$} & ${ }^{11} \mathrm{~B}\left(3 / 2^{-}\right)$ & $1 / 2$ & 1.69 & 1.81 & 1.301 & $0.74(19)^{\mathrm{m}}$ \\
\hline & & $3 / 2$ & 0.17 & 0.07 & 0.179 & $0.271(69)^{\mathrm{m}}$ \\
\hline & & sum & 1.76 & 1.88 & 1.48 & $1.20(26)^{\mathrm{m}}$ \\
\hline${ }^{12} \mathrm{C}\left(0^{+}\right)$ & ${ }^{11} \mathrm{~B}\left(3 / 2^{-}\right)$ & $3 / 2$ & 179.8 & 219.0 & 194.5 & $223(31)^{\mathrm{a}}$ \\
\hline \multirow[t]{3}{*}{${ }^{12} \mathrm{C}\left(2^{+}\right)$} & ${ }^{11} \mathrm{~B}\left(3 / 2^{-}\right)$ & $1 / 2$ & 18.52 & 19.71 & 25.07 & \\
\hline & & $3 / 2$ & 0.89 & 0.05 & 0.48 & \\
\hline & & sum & 19.41 & 19.76 & 25.55 & $15.8(35)^{\mathrm{a}}$ \\
\hline \multirow[t]{3}{*}{${ }^{12} \mathrm{~N}\left(1^{+}\right)$} & ${ }^{11} \mathrm{~B}\left(3 / 2^{-}\right)$ & $1 / 2$ & 2.47 & 2.64 & 1.92 & $1.4(2)^{\mathrm{n}}$ \\
\hline & & $3 / 2$ & 0.264 & 0.11 & 0.271 & $0.33(5)^{\mathrm{n}}$ \\
\hline & & sum & 2.73 & 2.75 & 2.19 & $1.73(25)^{\mathrm{n}} ; 1.63(25)^{\mathrm{o}}$ \\
\hline${ }^{11} \mathrm{~B}\left(3 / 2_{1}^{-}\right)$ & ${ }^{10} \mathrm{~B}\left(3^{+}\right)$ & $3 / 2$ & 14.12 & 15.84 & 13.08 & $31.6(18)^{\mathrm{h}}$ \\
\hline \multirow[t]{3}{*}{${ }^{11} \mathrm{~B}\left(3 / 2_{1}^{-}\right)$} & ${ }^{10} \mathrm{~B}\left(1^{+}\right)$ & $1 / 2$ & 2.20 & 3.509 & 2.061 & \\
\hline & & $3 / 2$ & 8.95 & 0.418 & 2.421 & \\
\hline & & sum & 11.15 & 3.927 & 4.482 & $14.9(18)^{\mathrm{h}}$ \\
\hline${ }^{11} \mathrm{C}\left(3 / 2_{1}^{-}\right)$ & ${ }^{10} \mathrm{~B}\left(3^{+}\right)$ & $3 / 2$ & 18.05 & 20.52 & 16.59 & $29(5)^{\mathrm{a}}$ \\
\hline${ }^{11} \mathrm{C}\left(3 / 2_{2}^{-}\right)$ & ${ }^{10} \mathrm{~B}\left(3^{+}\right)$ & $3 / 2$ & 0.141 & 0.066 & 0.105 & $0.107(10)^{\mathrm{a}}$ \\
\hline${ }^{10} \mathrm{~B}\left(3_{1}^{+}\right)$ & ${ }^{9} \mathrm{Be}\left(3 / 2^{-}\right)$ & $3 / 2$ & 3.31 & 3.51 & 3.53 & $5.26(37)^{\mathrm{a}} ; 5.06(46)^{\mathrm{p}}$ \\
\hline \multirow[t]{3}{*}{${ }^{10} \mathrm{~B}\left(1_{1}^{+}\right)$} & ${ }^{9} \mathrm{Be}\left(3 / 2^{-}\right)$ & $1 / 2$ & 0.003 & 3.37 & 3.09 & $5.50(41)^{\mathrm{a}} ; 1.27(21)^{\mathrm{p}}$ \\
\hline & & $3 / 2$ & 2.06 & 1.14 & 2.48 & $2.98(30)^{\mathrm{a}} ; 3.43(42)^{\mathrm{p}}$ \\
\hline & & sum & 2.06 & 4.51 & 5.57 & $8.43(71)^{\mathrm{a}} ; 4.70(63)^{\mathrm{p}}$ \\
\hline${ }^{10} \mathrm{~B}\left(0_{1}^{+}\right)$ & ${ }^{9} \mathrm{Be}\left(3 / 2^{-}\right)$ & $3 / 2$ & 5.03 & 5.81 & 5.36 & $8.0(6)^{\mathrm{a}} ; 4.35(59)^{\mathrm{p}}$ \\
\hline \multirow{3}{*}{${ }^{10} \mathrm{~B}\left(1_{2}^{+}\right)$} & ${ }^{9} \mathrm{Be}\left(3 / 2^{-}\right)$ & $1 / 2$ & 2.69 & 0.032 & 0.362 & $0.29(6)^{p}$ \\
\hline & & $3 / 2$ & 0.95 & 1.40 & 0.713 & $0.82(12)^{\mathrm{p}}$ \\
\hline & & sum & 3.64 & 1.73 & 1.075 & $1.46(17)^{\mathrm{a}} ; 1.11(18)^{\mathrm{p}}$ \\
\hline${ }^{10} \mathrm{~B}\left(3_{1}^{+}\right)$ & ${ }^{9} \mathrm{~B}\left(3 / 2^{-}\right)$ & $3 / 2$ & 2.56 & 2.71 & 2.18 & $1.93(29)^{\mathrm{h}}$ \\
\hline \multirow[t]{3}{*}{${ }^{10} \mathrm{~B}\left(3_{1}^{+}\right)$} & ${ }^{9} \mathrm{~B}\left(5 / 2^{-}\right)$ & $1 / 2$ & 0.39 & 0.73 & 0.71 & \\
\hline & & $3 / 2$ & 3.43 & 4.324 & 4.23 & \\
\hline & & sum & 3.82 & 5.05 & 4.94 & $2.15(29)^{\mathrm{h}}$ \\
\hline \multirow[t]{3}{*}{${ }^{9} \operatorname{Li}\left(3 / 2^{-}\right)$} & ${ }^{8} \mathrm{Li}\left(2^{+}\right)$ & $1 / 2$ & 0.017 & 0.060 & 0.052 & \\
\hline & & $3 / 2$ & 1.011 & 1.224 & 1.135 & \\
\hline & & sum & 1.028 & 1.284 & 1.187 & $1.33(33)^{\mathrm{q}}$ \\
\hline${ }^{9} \mathrm{Be}\left(3 / 2^{-}\right)$ & ${ }^{8} \mathrm{Be}\left(0^{+}\right)$ & $3 / 2$ & 0.166 & 0.157 & 0.166 & $0.27(9)^{\mathrm{h}}$ \\
\hline${ }^{9} \mathrm{Be}\left(3 / 2^{-}\right)$ & ${ }^{8} \mathrm{Be}\left(2^{+}\right)$ & $1 / 2$ & 0.088 & 0.085 & 0.097 & \\
\hline & & $3 / 2$ & 1.19 & 1.49 & 1.23 & \\
\hline & & sum & 1.28 & 1.58 & 1.33 & $2.0(11)^{\mathrm{h}}$ \\
\hline
\end{tabular}


TABLE VI. (Continued.)

\begin{tabular}{|c|c|c|c|c|c|c|}
\hline \multirow[t]{2}{*}{$A$} & \multirow[t]{2}{*}{$A-1$} & \multirow[t]{2}{*}{$j$} & \multicolumn{3}{|c|}{$C_{l j}^{2}$} & \multirow[t]{2}{*}{$C_{\text {exp }}^{2}$} \\
\hline & & & Boyarkina & CK816 & Millener & \\
\hline \multirow[t]{3}{*}{${ }^{9} \mathrm{C}\left(3 / 2^{-}\right)$} & \multirow[t]{3}{*}{${ }^{8} \mathrm{~B}\left(2^{+}\right)$} & $1 / 2$ & 0.012 & 0.057 & 0.051 & \\
\hline & & $3 / 2$ & 0.990 & 1.138 & 1.068 & \\
\hline & & sum & 1.002 & 1.195 & 1.119 & $1.22(13)^{\mathrm{r}} ; 1.18(34){ }^{\mathrm{s}} 1.27(10)^{\mathrm{t}}$ \\
\hline \multirow[t]{2}{*}{${ }^{8} \operatorname{Li}\left(2_{1}^{+}\right)$} & \multirow[t]{2}{*}{${ }^{7} \operatorname{Li}\left(3 / 2^{-}\right)$} & $1 / 2$ & 0.046 & 0.011 & 0.030 & $0.048(6)^{\mathrm{u}}$ \\
\hline & & $3 / 2$ & 0.271 & 0.326 & 0.324 & $0.384(38)^{\mathrm{u}}$ \\
\hline \multirow{2}{*}{${ }^{8} \operatorname{Li}\left(1_{1}^{+}\right)$} & \multirow{2}{*}{${ }^{7} \operatorname{Li}\left(3 / 2^{-}\right)$} & $1 / 2$ & 0.139 & 0.044 & 0.019 & $0.015(2)^{\mathrm{u}}$ \\
\hline & & $3 / 2$ & 0.038 & 0.028 & 0.056 & $0.068(13)^{\mathrm{u}}$ \\
\hline \multirow[t]{2}{*}{${ }^{8} \mathrm{~B}\left(2_{1}^{+}\right)$} & \multirow[t]{2}{*}{${ }^{7} \mathrm{Be}\left(3 / 2^{-}\right)$} & $1 / 2$ & 0.055 & 0.012 & 0.035 & $0.062(10)^{\mathrm{v}} ; 0.058(8)^{\mathrm{w}}$ \\
\hline & & $3 / 2$ & 0.334 & 0.399 & 0.396 & $0.398(62)^{\mathrm{v}} ; 0.371(43)^{\mathrm{w}}$ \\
\hline \multirow[t]{3}{*}{${ }^{7} \operatorname{Li}\left(3 / 2^{-}\right)$} & \multirow[t]{3}{*}{${ }^{6} \operatorname{Li}\left(1^{+}\right)$} & $1 / 2$ & 1.05 & 1.09 & 1.04 & \\
\hline & & $3 / 2$ & 2.11 & 1.52 & 1.90 & \\
\hline & & sum & 3.15 & 2.61 & 2.94 & $3.17(53)^{\mathrm{h}}$ \\
\hline${ }^{7} \mathrm{Li}\left(3 / 2^{-}\right)$ & ${ }^{6} \mathrm{Li}\left(3^{+}\right)$ & $3 / 2$ & 4.45 & 4.56 & 4.52 & $4.24(48)^{\mathrm{h}}$ \\
\hline${ }^{7} \mathrm{Li}\left(3 / 2^{-}\right)$ & ${ }^{6} \mathrm{Li}\left(0^{+}\right)$ & $3 / 2$ & 2.42 & 2.57 & 2.47 & $2.91(35)^{\mathrm{h}}$ \\
\hline
\end{tabular}

${ }^{\mathrm{a}}$ From the $\left({ }^{3} \mathrm{He}, d\right)$ compilation of Ref. [34].

${ }^{\mathrm{b}}$ This is a corrected value of the ANC from Ref. [41] based on the updated value of the ANC for ${ }^{3} \mathrm{He}$.

${ }^{c}$ From a zero-range DWBA analysis of ${ }^{13} \mathrm{C}\left({ }^{3} \mathrm{He}, d\right){ }^{14} \mathrm{~N}$ [47].

${ }^{\mathrm{d}}$ From $\left({ }^{3} \mathrm{He}, d\right)[36]$.

${ }^{\text {e From }}\left({ }^{3} \mathrm{He}, d\right)[49]$. Corrected ANCs are shown according to updated value of the ${ }^{3} \mathrm{He}$ ANC.

${ }^{\mathrm{f}}$ From ${ }^{13} \mathrm{C}\left({ }^{14} \mathrm{~N},{ }^{13} \mathrm{C}\right){ }^{14} \mathrm{~N}[50]$.

${ }^{\mathrm{g}} C_{p 3 / 2}=0$ was assumed in the analysis of the transfer to this state.

${ }^{\text {h}}$ From the $(d, t)$ reaction at $E_{d}=18 \mathrm{MeV}$ [51].

${ }^{\mathrm{i}}$ From ${ }^{14} \mathrm{~N}\left({ }^{13} \mathrm{~N},{ }^{14} \mathrm{O}\right){ }^{13} \mathrm{C}[52]$.

${ }^{\mathrm{j}}$ From $(d, n)[53]$.

${ }^{\mathrm{k}}$ From ${ }^{12} \mathrm{C}\left({ }^{13} \mathrm{C},{ }^{12} \mathrm{C}\right){ }^{13} \mathrm{C}[54]$.

${ }^{1}$ From ${ }^{14} \mathrm{~N}\left({ }^{12} \mathrm{~N},{ }^{13} \mathrm{O}\right){ }^{13} \mathrm{C}[55]$.

${ }^{\mathrm{m}}$ This value has been obtained in Ref. [56] from analysis of ${ }^{11} \mathrm{~B}(d, p)^{12} \mathrm{~B}$ using adiabatic theory. The DWBA gives a larger value, 1.34(23) [57]. However, the same DWBA analysis of Ref. [57] overestimates the ${ }^{13} \mathrm{C}$ ANC by $55 \%$.

${ }^{n}$ From ${ }^{14} \mathrm{~N}\left({ }^{11} \mathrm{C},{ }^{12} \mathrm{~N}\right){ }^{13} \mathrm{C}[58]$.

${ }^{\circ}$ The ${ }^{12} \mathrm{~N}$ ANC is obtained using relations between ANCs in mirror systems [37] and the ${ }^{12} \mathrm{~B}$ ANC from [56].

${ }^{\mathrm{p}}$ From ${ }^{9} \mathrm{Be}\left({ }^{10} \mathrm{~B},{ }^{9} \mathrm{Be}\right){ }^{10} \mathrm{~B}[59]$.

${ }^{\mathrm{q}} \mathrm{From}{ }^{8} \operatorname{Li}(d, p){ }^{9} \operatorname{Li}[60]$.

${ }^{\mathrm{r}}$ From one-proton removal of ${ }^{9} \mathrm{C}[61]$.

${ }^{\mathrm{s}}$ From $d\left({ }^{8} \mathrm{~B},{ }^{9} \mathrm{C}\right) n[62]$.

${ }^{\mathrm{t}}$ From proton knockout on ${ }^{9} \mathrm{C}$ [63].

${ }^{\text {uFrom }}{ }^{13} \mathrm{C}\left({ }^{7} \mathrm{Li},{ }^{8} \mathrm{Li}\right){ }^{12} \mathrm{C}[64]$.

${ }^{\mathrm{v}}$ From ${ }^{10} \mathrm{~B}\left({ }^{7} \mathrm{Be},{ }^{8} \mathrm{~B}\right){ }^{9} \mathrm{Be}[65] .\left(C_{p 1 / 2} / C_{p 3 / 2}\right)^{2}=0.157$ was fixed in the analysis.

${ }^{\mathrm{w}}$ From ${ }^{14} \mathrm{~N}\left({ }^{7} \mathrm{Be},{ }^{8} \mathrm{~B}\right){ }^{13} \mathrm{C}[66] .\left(C_{p 1 / 2} / C_{p 3 / 2}\right)^{2}=0.157$ was fixed in the analysis.

\section{C. $A=13$}

The overlap $\left\langle{ }^{13} \mathrm{C} \mid{ }^{12} \mathrm{C}\right\rangle$ and its mirror analog $\left\langle{ }^{13} \mathrm{~N} \mid{ }^{12} \mathrm{C}\right\rangle$ are very sensitive both to the choice of the shell-model interaction for ${ }^{13} \mathrm{C}$ and ${ }^{12} \mathrm{C}$ nuclei and to the spin-orbit and tensor parts of the effective interaction $\tilde{V}^{\text {eff }}$. By tuning them, it is possible to reach a good agreement with the experimental value for the ${ }^{13} \mathrm{C}$ and ${ }^{13} \mathrm{~N}$ ANCs. The other overlap involving an $A=13$ nucleus, $\left\langle{ }^{13} \mathrm{O} \mid{ }^{12} \mathrm{~N}\right\rangle$, is not very sensitive to the choice of the shell-model interaction for ${ }^{13} \mathrm{O}$ and ${ }^{12} \mathrm{~N}$ wave functions. The predicted ANC for this overlap is larger than $C_{\text {exp }}$. However, $C_{\text {exp }}$ has been obtained by assuming that no contribution from $j=3 / 2$ is present in the experimental cross sections. This contribution could be non-negligible, as $C_{p 3 / 2}^{2}$ is $\sim 10 \%$ of $C_{p 1 / 2}^{2}$, and thus it influences the ANC obtained.
For all the $A=13$ nuclei considered, the difference between $S_{l j}^{\mathrm{IE}}$ and $S_{l j}^{\mathrm{DE}}$ depends on $j$. For $j=1 / 2, S_{l j}^{\mathrm{IE}}$ is slightly larger than $S_{l j}^{\mathrm{DE}}$, except for the strongly bound proton in ${ }^{13} \mathrm{C}$, whereas for $j=3 / 2 S_{l j}^{\mathrm{IE}}$ is significantly reduced from $S_{l j}^{\mathrm{DE}}$. The $\mathrm{SF}$ for $\left\langle{ }^{13} \mathrm{C} \mid{ }^{12} \mathrm{C}\right\rangle$ is larger than $S_{\text {exp }}$ but its ANC is lower than $C_{\text {exp. }}$. This means that the current calculations do not correctly give the shape of this overlap, in particular, its rms radius.

\section{D. $A=12$}

For the astrophysically relevant overlap $\left\langle{ }^{12} \mathrm{~N} \mid{ }^{11} \mathrm{C}\right\rangle$, both the ANCs and the $C_{p 3 / 2} / C_{p 1 / 2}$ ratio depend on the choice of the shell-model interaction. The Millener interaction gives the best result for the ANC, although it is still overestimated 
TABLE VII. The squared ANCs, $C_{l j}^{2}$ (in $\mathrm{fm}^{-1}$ ), rms radii $\left\langle r^{2}\right\rangle^{1 / 2}$ (in fm), and the SFs $S_{l j}^{\mathrm{IE}}$ and $S_{l j}^{\mathrm{DE}}$ for a range of the $\langle A \mid A-1\rangle$ overlaps calculated with the M3YE potential in comparison to $S_{\exp }$ derived experimentally and $S_{a b}$ from ab initio calculations.

\begin{tabular}{|c|c|c|c|c|c|c|c|c|}
\hline$A$ & $A-1$ & $j$ & $C_{l j}^{2}$ & $\left\langle r^{2}\right\rangle^{1 / 2}$ & $S_{l j}^{\mathrm{IE}}$ & $S_{l j}^{\mathrm{DE}}$ & $S_{a b}$ & $S_{\text {exp }}$ \\
\hline${ }^{15} \mathrm{~N}\left(1 / 2^{-}\right)$ & ${ }^{14} \mathrm{C}\left(0^{+}\right)$ & $1 / 2$ & 53.22 & 2.968 & 0.832 & 0.997 & & \\
\hline \multirow[t]{3}{*}{${ }^{15} \mathrm{~N}\left(1 / 2^{-}\right)$} & ${ }^{14} \mathrm{~N}\left(1^{+}\right)$ & $1 / 2$ & 32.75 & 3.013 & 1.22 & 1.36 & & \\
\hline & & $3 / 2$ & 1.33 & 2.614 & 0.088 & 0.122 & & \\
\hline & & sum & & & 1.31 & 1.48 & & $0.93(15)^{\mathrm{a}}$ \\
\hline \multirow[t]{2}{*}{${ }^{15} \mathrm{O}\left(1 / 2^{-}\right)$} & ${ }^{14} \mathrm{~N}\left(1^{+}\right)$ & $1 / 2$ & 50.44 & 3.081 & 1.31 & 1.36 & & \\
\hline & & $3 / 2$ & 2.07 & 2.687 & 0.091 & 0.122 & & \\
\hline \multirow[t]{2}{*}{${ }^{15} \mathrm{O}\left(3 / 2^{-}\right)$} & ${ }^{14} \mathrm{~N}\left(1^{+}\right)$ & $1 / 2$ & 0.511 & 3.963 & 0.090 & 0.061 & & \\
\hline & & $3 / 2$ & $5.7 \times 10^{-6}$ & 3.225 & $4.0 \times 10^{-4}$ & $1.5 \times 10^{-3}$ & & \\
\hline${ }^{14} \mathrm{C}\left(0^{+}\right)$ & ${ }^{13} \mathrm{~B}\left(3 / 2^{-}\right)$ & $3 / 2$ & 762.5 & 2.656 & 2.107 & 4.047 & & \\
\hline${ }^{14} \mathrm{C}\left(0^{+}\right)$ & ${ }^{13} \mathrm{C}\left(1 / 2^{-}\right)$ & $1 / 2$ & 21.37 & 3.099 & 1.807 & 1.98 & & $1.07(22)^{\mathrm{a}}$ \\
\hline \multirow[t]{2}{*}{${ }^{14} \mathrm{~N}\left(1_{1}^{+}\right)$} & ${ }^{13} \mathrm{C}\left(1 / 2^{-}\right)$ & $1 / 2$ & 18.5 & 3.024 & 0.628 & 0.705 & & \\
\hline & & $3 / 2$ & 0.25 & 3.203 & 0.0073 & 0.0125 & & \\
\hline${ }^{14} \mathrm{~N}\left(0^{+}\right)$ & ${ }^{13} \mathrm{C}\left(1 / 2^{-}\right)$ & $1 / 2$ & 13.6 & 3.193 & 0.858 & 0.933 & & \\
\hline \multirow{2}{*}{${ }^{14} \mathrm{~N}\left(1_{2}^{+}\right)$} & ${ }^{13} \mathrm{C}\left(1 / 2^{-}\right)$ & $1 / 2$ & 1.525 & 3.415 & 0.150 & 0.130 & & \\
\hline & & $3 / 2$ & 0.596 & 3.039 & 0.097 & 0.144 & & \\
\hline${ }^{14} \mathrm{~N}\left(2^{+}\right)$ & ${ }^{13} \mathrm{C}\left(1 / 2^{-}\right)$ & $3 / 2$ & 0.071 & 3.639 & 0.040 & 0.051 & & \\
\hline \multirow[t]{2}{*}{${ }^{14} \mathrm{~N}\left(1_{1}^{+}\right)$} & ${ }^{13} \mathrm{~N}\left(1 / 2^{-}\right)$ & $1 / 2$ & 12.7 & 2.965 & 0.589 & 0.704 & & $0.48(8)^{\mathrm{a}}$ \\
\hline & & $3 / 2$ & 0.161 & 3.157 & 0.0064 & 0.0124 & & \\
\hline${ }^{14} \mathrm{O}\left(0^{+}\right)$ & ${ }^{13} \mathrm{~N}\left(1 / 2^{-}\right)$ & $1 / 2$ & 31.9 & 3.206 & 1.87 & 1.98 & & \\
\hline${ }^{14} \mathrm{O}\left(0^{+}\right)$ & ${ }^{13} \mathrm{O}\left(3 / 2^{-}\right)$ & $3 / 2$ & 547 & 2.642 & 2.08 & 4.05 & & \\
\hline \multirow{2}{*}{${ }^{13} \mathrm{C}\left(1 / 2^{-}\right)$} & ${ }^{12} \mathrm{~B}\left(1^{+}\right)$ & $1 / 2$ & 2.420 & 2.749 & 0.011 & 0.014 & & \\
\hline & & $3 / 2$ & 103.6 & 2.608 & 0.675 & 1.197 & & \\
\hline${ }^{13} \mathrm{C}\left(1 / 2^{-}\right)$ & ${ }^{12} \mathrm{C}\left(0^{+}\right)$ & $1 / 2$ & 2.067 & 3.226 & 0.642 & 0.633 & & $0.54(7)^{\mathrm{a}}$ \\
\hline${ }^{13} \mathrm{C}\left(1 / 2^{-}\right)$ & ${ }^{12} \mathrm{C}\left(2^{+}\right)$ & $1 / 2$ & 6.597 & 2.762 & 0.6195 & 1.116 & & \\
\hline${ }^{13} \mathrm{~N}\left(1 / 2^{-}\right)$ & ${ }^{12} \mathrm{C}\left(0^{+}\right)$ & $1 / 2$ & 2.625 & 3.432 & 0.727 & 0.633 & & \\
\hline \multirow[t]{2}{*}{${ }^{13} \mathrm{O}\left(3 / 2^{-}\right)$} & ${ }^{12} \mathrm{~N}\left(1^{+}\right)$ & $1 / 2$ & 3.254 & 3.508 & 0.738 & 0.595 & & \\
\hline & & $3 / 2$ & 0.361 & 3.237 & 0.107 & 0.126 & & \\
\hline \multirow[t]{3}{*}{${ }^{12} \mathrm{~B}\left(1^{+}\right)$} & ${ }^{11} \mathrm{~B}\left(3 / 2^{-}\right)$ & $1 / 2$ & 1.301 & 3.455 & 0.834 & 0.7832 & & \\
\hline & & $3 / 2$ & 0.179 & 3.287 & 0.134 & 0.205 & & \\
\hline & & sum & & & 0.968 & 0.988 & & $0.40(6)^{\mathrm{a}}$ \\
\hline${ }^{12} \mathrm{C}\left(0^{+}\right)$ & ${ }^{11} \mathrm{~B}\left(3 / 2^{-}\right)$ & $3 / 2$ & 194.5 & 2.687 & 1.550 & 2.8546 & & $1.72(11)^{b}$ \\
\hline${ }^{12} \mathrm{C}\left(0^{+}\right)$ & ${ }^{11} \mathrm{C}\left(3 / 2^{-}\right)$ & $3 / 2$ & 148.3 & 2.663 & 1.496 & 2.8546 & & $2.16(25)^{\mathrm{a}}$ \\
\hline \multirow[t]{2}{*}{${ }^{12} \mathrm{C}\left(2^{+}\right)$} & ${ }^{11} \mathrm{~B}\left(3 / 2^{-}\right)$ & $1 / 2$ & 25.07 & 2.859 & 0.435 & 0.598 & & \\
\hline & & $3 / 2$ & 0.478 & 2.859 & 0.009 & 0.013 & & \\
\hline \multirow[t]{2}{*}{${ }^{12} \mathrm{~N}\left(1^{+}\right)$} & ${ }^{11} \mathrm{C}\left(3 / 2^{-}\right)$ & $1 / 2$ & 1.915 & 3.847 & 0.9647 & 0.7832 & & \\
\hline & & $3 / 2$ & 0.156 & 3.669 & 0.271 & 0.205 & & \\
\hline${ }^{11} \mathrm{~B}\left(3 / 2_{1}^{-}\right)$ & ${ }^{10} \mathrm{~B}\left(3^{+}\right)$ & $3 / 2$ & 13.08 & 2.814 & 0.611 & 1.105 & & \\
\hline \multirow[t]{2}{*}{${ }^{11} \mathrm{~B}\left(3 / 2_{1}^{-}\right)$} & ${ }^{10} \mathrm{~B}\left(1^{+}\right)$ & $1 / 2$ & 2.061 & 2.891 & 0.067 & 0.106 & & \\
\hline & & $3 / 2$ & 2.421 & 2.758 & 0.104 & 0.182 & & \\
\hline${ }^{11} \mathrm{C}\left(3 / 2_{1}^{-}\right)$ & ${ }^{10} \mathrm{~B}\left(3^{+}\right)$ & $3 / 2$ & 16.59 & 2.864 & 0.645 & 1.105 & & \\
\hline${ }^{11} \mathrm{C}\left(3 / 2_{2}^{-}\right)$ & ${ }^{10} \mathrm{~B}\left(3^{+}\right)$ & $3 / 2$ & 0.105 & 3.298 & 0.015 & 0.021 & & \\
\hline${ }^{10} \mathrm{Be}\left(0^{+}\right)$ & ${ }^{9} \mathrm{Li}\left(3 / 2^{-}\right)$ & $3 / 2$ & 171.9 & 2.723 & 0.819 & 1.931 & $1.043^{\mathrm{c}}$ & \\
\hline${ }^{10} \mathrm{Be}\left(0^{+}\right)$ & ${ }^{9} \mathrm{Be}\left(3 / 2^{-}\right)$ & $3 / 2$ & 8.83 & 3.035 & 1.503 & 2.672 & $1.932^{\mathrm{c}}$ & \\
\hline${ }^{10} \mathrm{~B}\left(3^{+}\right)$ & ${ }^{9} \mathrm{Be}\left(3 / 2^{-}\right)$ & $3 / 2$ & 3.53 & 2.940 & 0.323 & 0.665 & & \\
\hline \multirow[t]{2}{*}{${ }^{10} \mathrm{~B}\left(1_{1}^{+}\right)$} & ${ }^{9} \mathrm{Be}\left(3 / 2^{-}\right)$ & $1 / 2$ & 3.09 & 3.151 & 0.290 & 0.432 & & \\
\hline & & $3 / 2$ & 2.48 & 3.039 & 0.255 & 0.506 & & \\
\hline${ }^{10} \mathrm{~B}\left(0^{+}\right)$ & ${ }^{9} \mathrm{Be}\left(3 / 2^{-}\right)$ & $3 / 2$ & 5.36 & 3.107 & 0.799 & 1.34 & & \\
\hline \multirow[t]{2}{*}{${ }^{10} \mathrm{~B}\left(1_{2}^{+}\right)$} & ${ }^{9} \mathrm{Be}\left(3 / 2^{-}\right)$ & $1 / 2$ & 0.362 & 3.255 & 0.059 & 0.081 & & \\
\hline & & $3 / 2$ & 0.713 & 3.085 & 0.131 & 0.252 & & \\
\hline${ }^{10} \mathrm{~B}\left(3^{+}\right)$ & ${ }^{9} \mathrm{~B}\left(3 / 2^{-}\right)$ & $3 / 2$ & 2.18 & 2.894 & 0.308 & 0.665 & & \\
\hline \multirow[t]{2}{*}{${ }^{10} \mathrm{~B}\left(3^{+}\right)$} & ${ }^{9} \mathrm{~B}\left(5 / 2^{-}\right)$ & $1 / 2$ & 0.719 & 2.991 & 0.031 & 0.068 & & \\
\hline & & $3 / 2$ & 4.23 & 2.86 & 0.226 & 0.683 & & \\
\hline${ }^{9} \mathrm{Li}\left(3 / 2^{-}\right)$ & ${ }^{8} \mathrm{He}\left(0^{+}\right)$ & $3 / 2$ & 21.39 & 2.810 & 0.385 & 0.935 & & \\
\hline${ }^{9} \mathrm{Li}\left(3 / 2^{-}\right)$ & ${ }^{8} \mathrm{Li}\left(2^{+}\right)$ & $1 / 2$ & 0.052 & 3.508 & 0.022 & 0.029 & $0.120^{\mathrm{c}}$ & \\
\hline
\end{tabular}


TABLE VII. (Continued.)

\begin{tabular}{|c|c|c|c|c|c|c|c|c|}
\hline$A$ & $A-1$ & $j$ & $C_{l j}^{2}$ & $\left\langle r^{2}\right\rangle^{1 / 2}$ & $S_{l j}^{\mathrm{IE}}$ & $S_{l j}^{\mathrm{DE}}$ & $S_{a b}$ & $S_{\text {exp }}$ \\
\hline & & $3 / 2$ & 1.135 & 3.282 & 0.578 & 1.016 & $1.02^{\mathrm{c}}$ & \\
\hline & & sum & & & 0.600 & 1.045 & $1.14^{\mathrm{c}}$ & $0.59(15)^{\mathrm{d}}$ \\
\hline \multirow[t]{3}{*}{${ }^{9} \operatorname{Li}\left(3 / 2^{-}\right)$} & ${ }^{8} \operatorname{Li}\left(1^{+}\right)$ & $1 / 2$ & 0.143 & 3.352 & 0.040 & 0.060 & $0.009^{c}$ & \\
\hline & & $3 / 2$ & 0.820 & 3.162 & 0.278 & 0.433 & $0.473^{c}$ & \\
\hline & & sum & & & 0.318 & 0.493 & $0.482^{\mathrm{c}}$ & $0.28(7)^{\mathrm{d}}$ \\
\hline${ }^{9} \mathrm{Li}\left(3 / 2^{-}\right)$ & ${ }^{8} \mathrm{Li}\left(3^{+}\right)$ & $3 / 2$ & 4.091 & 3.126 & 0.782 & 1.512 & $0.951^{\mathrm{c}}$ & $0.94(20)^{\mathrm{d}}$ \\
\hline \multirow[t]{2}{*}{${ }^{9} \mathrm{Be}\left(3 / 2^{-}\right)$} & ${ }^{8} \operatorname{Li}\left(2^{+}\right)$ & $1 / 2$ & 7.904 & 2.844 & 0.057 & 0.126 & $0.154^{\mathrm{c}}$ & \\
\hline & & $3 / 2$ & 51.53 & 2.788 & 0.406 & 1.000 & $0.575^{\mathrm{c}}$ & \\
\hline${ }^{9} \mathrm{Be}\left(3 / 2^{-}\right)$ & ${ }^{8} \mathrm{Be}\left(0^{+}\right)$ & $3 / 2$ & 0.166 & 3.760 & 0.404 & 0.6274 & & \\
\hline \multirow[t]{2}{*}{${ }^{9} \mathrm{Be}\left(3 / 2^{-}\right)$} & ${ }^{8} \mathrm{Be}\left(2^{+}\right)$ & $1 / 2$ & 0.097 & 3.425 & 0.030 & 0.042 & & \\
\hline & & $3 / 2$ & 12.3 & 3.145 & 0.461 & 0.817 & & \\
\hline \multirow[t]{3}{*}{${ }^{9} \mathrm{C}\left(3 / 2^{-}\right)$} & ${ }^{8} \mathrm{~B}\left(2^{+}\right)$ & $1 / 2$ & 0.051 & 3.834 & 0.027 & 0.029 & & \\
\hline & & $3 / 2$ & 1.068 & 3.597 & 0.6811 & 1.016 & & \\
\hline & & sum & & & 0.708 & 1.045 & & $0.77(6)^{\mathrm{e}}$ \\
\hline${ }^{9} \mathrm{C}\left(3 / 2^{-}\right)$ & ${ }^{8} \mathrm{C}\left(0^{+}\right)$ & $3 / 2$ & 17.24 & 2.814 & 0.397 & 0.935 & & \\
\hline \multirow[t]{3}{*}{${ }^{8} \mathrm{Li}\left(2^{+}\right)$} & ${ }^{7} \mathrm{He}\left(3 / 2^{-}\right)$ & $1 / 2$ & 3.10 & 2.943 & 0.070 & 0.146 & & \\
\hline & & $3 / 2$ & 13.2 & 3.157 & 0.333 & 0.785 & & \\
\hline & & sum & & & 0.403 & 0.931 & $0.58^{f}$ & $0.36(7)^{\mathrm{f}}$ \\
\hline \multirow[t]{3}{*}{${ }^{8} \operatorname{Li}\left(2^{+}\right)$} & ${ }^{7} \mathrm{He}\left(5 / 2^{-}\right)$ & $1 / 2$ & 2.98 & 2.878 & 0.030 & 0.060 & & \\
\hline & & $3 / 2$ & 5.42 & 2.855 & 0.067 & 0.139 & & \\
\hline & & sum & & & 0.097 & 0.199 & $0.17^{f}$ & $0.29(15)^{\mathrm{f}}$ \\
\hline \multirow[t]{2}{*}{${ }^{8} \mathrm{Li}\left(2^{+}\right)$} & ${ }^{7} \operatorname{Li}\left(3 / 2^{-}\right)$ & $1 / 2$ & 0.030 & 3.837 & 0.046 & 0.064 & $0.082^{\mathrm{c}}$ & \\
\hline & & $3 / 2$ & 0.324 & 3.620 & 0.605 & 1.079 & $0.884^{\mathrm{c}}$ & \\
\hline \multirow[t]{2}{*}{${ }^{8} \operatorname{Li}\left(1^{+}\right)$} & ${ }^{7} \mathrm{Li}\left(3 / 2^{-}\right)$ & $1 / 2$ & 0.019 & 4.331 & 0.081 & 0.100 & & \\
\hline & & $3 / 2$ & 0.056 & 4.137 & 0.268 & 0.417 & & \\
\hline${ }^{8} \mathrm{Be}\left(0^{+}\right)$ & ${ }^{7} \mathrm{Li}\left(3 / 2^{-}\right)$ & $3 / 2$ & 88.1 & 2.794 & 0.673 & 1.721 & & \\
\hline \multirow[t]{3}{*}{${ }^{8} \mathrm{~B}\left(2^{+}\right)$} & ${ }^{7} \mathrm{Be}\left(3 / 2^{-}\right)$ & $1 / 2$ & 0.035 & 4.659 & 0.055 & 0.064 & & \\
\hline & & $3 / 2$ & 0.396 & 4.397 & 0.728 & 1.079 & & \\
\hline & & sum & & & 0.783 & 1.143 & & $0.89(7)^{\mathrm{e}}$ \\
\hline${ }^{7} \mathrm{Li}\left(3 / 2^{-}\right)$ & ${ }^{6} \mathrm{He}\left(0^{+}\right)$ & $3 / 2$ & 5.62 & 2.871 & 0.284 & 0.693 & $0.439^{\mathrm{c}}$ & $0.44(6)^{\mathrm{f}} ; 0.42(4)^{\mathrm{g}}$ \\
\hline \multirow[t]{3}{*}{${ }^{7} \mathrm{Li}\left(3 / 2^{-}\right)$} & ${ }^{6} \mathrm{He}\left(2^{+}\right)$ & $1 / 2$ & 3.35 & 3.012 & 0.089 & 0.189 & $0.137^{\mathrm{c}}$ & \\
\hline & & $3 / 2$ & 3.93 & 2.945 & 0.115 & 0.255 & $0.156^{\mathrm{c}}$ & \\
\hline & & sum & & & 0.204 & 0.444 & 0.293 & $0.16(2)^{\mathrm{g}}$ \\
\hline \multirow[t]{3}{*}{${ }^{7} \operatorname{Li}\left(3 / 2^{-}\right)$} & ${ }^{6} \mathrm{Li}\left(1^{+}\right)$ & $1 / 2$ & 1.04 & 3.175 & 0.135 & 0.284 & $0.242^{\mathrm{c}}$ & \\
\hline & & $3 / 2$ & 1.90 & 3.064 & 0.301 & 0.587 & $0.473^{\mathrm{c}}$ & \\
\hline & & sum & & & 0.436 & 0.871 & 0.715 & $0.74(11)^{\mathrm{f}}$ \\
\hline${ }^{7} \mathrm{Li}\left(3 / 2^{-}\right)$ & ${ }^{6} \mathrm{Li}\left(3^{+}\right)$ & $3 / 2$ & 4.52 & 3.027 & 0.333 & 0.698 & $0.476^{\mathrm{c}}$ & \\
\hline${ }^{7} \mathrm{Li}\left(3 / 2^{-}\right)$ & ${ }^{6} \mathrm{Li}\left(0^{+}\right)$ & $3 / 2$ & 2.47 & 2.915 & 0.142 & 0.346 & & $0.19(3)^{\mathrm{f}}$ \\
\hline
\end{tabular}

${ }^{\mathrm{a}}$ From analysis of $(p, d)$ and $(d, p)$ cross sections using adiabatic theory and Hartree-Fock geometry for the transferred neutron potential well [3].

${ }^{\mathrm{b}}$ From ${ }^{12} \mathrm{C}\left(e, e^{\prime} p\right){ }^{11} \mathrm{~B}$ knockout [2].

${ }^{\mathrm{c}} \mathrm{VMC}$ calculations from Ref. [69].

${ }^{\mathrm{d}}$ From ${ }^{9} \mathrm{Li}(d, t){ }^{8} \mathrm{Li}$ where VMC overlap has been used for the transferred nucleon [67].

${ }^{\mathrm{e}}$ From proton knockout [63].

${ }^{\mathrm{f}}$ From Ref. [68].

${ }^{\mathrm{g}} \mathrm{From}{ }^{7} \mathrm{Li}\left(e, e^{\prime} p\right){ }^{6} \mathrm{He}[7]$.

in the $j=1 / 2$ channel. This overestimation correlates with enhancement of $S^{\mathrm{IE}}$ with respect to $S^{\mathrm{DE}}$. For the mirror overlap $\left\langle{ }^{12} \mathrm{~B} \mid{ }^{11} \mathrm{~B}\right\rangle$, where the experimental $\mathrm{SF}$ is available, $S^{\mathrm{IE}}$ is twice $S_{\text {exp }}$. Renormalization of $\tilde{V}^{\text {eff }}$ from Ref. [11] can lower the ANC close to $C_{\text {exp }}$ but it does not change $S^{\mathrm{IE}}$ to the same extent.

For the overlap $\left\langle{ }^{12} \mathrm{C} \mid{ }^{11} \mathrm{~B}\right\rangle$, the ANC with the Millener interaction is slightly smaller than $C_{\exp }$ and the $\mathrm{SF} S^{\mathrm{IE}}$ is smaller than $S_{\exp }$ to the same extent. However, the rms radius of this overlap, $2.68 \mathrm{fm}$, is smaller than the values of $2.780(16) \mathrm{fm}$ fitted to the ${ }^{12} \mathrm{C}\left(e, e^{\prime} p\right){ }^{11} \mathrm{~B}$ data. The $\mathrm{SF}$ $S^{\mathrm{IE}}$ is reduced with respect to $S^{\mathrm{DE}}$. The latter, equal to 2.85 , increases to 3.19 if the shell-model space is enlarged to $4 \hbar \omega$ [4], in disagreement both with the $\left(e, e^{\prime} p\right)$ and the proton knockout cross sections. It is possible to bring both the $S^{\mathrm{IE}}$ and the ANC into agreement with experimental values by tuning $\tilde{V}^{\text {eff }}$. It should be noted, however, that $S_{\exp }$ for the mirror overlap, $\left\langle{ }^{12} \mathrm{C} \mid{ }^{11} \mathrm{C}\right\rangle$, determined from the $(d, p)$ 
reaction does not agree with the one obtained from the $\left(e, e^{\prime} p\right)$ analysis.

The ANC for the $\left\langle{ }^{12} \mathrm{C}\left(2^{+}\right) \mid{ }^{11} \mathrm{~B}\right\rangle$ overlap is available from the $\left({ }^{3} \mathrm{He}, d\right)$ reaction [34]. The present calculations give a much larger value for this ANC while keeping the $\mathrm{SF} S^{\mathrm{IE}}$ reduced.

\section{E. $A=11$}

The SF reduction in the overlaps $\left\langle{ }^{11} \mathrm{~B} \mid{ }^{10} \mathrm{~B}\right\rangle$ and $\left\langle{ }^{11} \mathrm{C} \mid{ }^{10} \mathrm{~B}\right\rangle$ is about $\sim 50 \%$ for all the states considered. Such a reduction is typical for large separation energies similar to those in ${ }^{11} \mathrm{~B}$ and ${ }^{11} \mathrm{C}$. In other nuclei such as ${ }^{16} \mathrm{O}$ and ${ }^{12} \mathrm{C}$, where a similar SF reduction occurs, the calculated ANCs would agree with $C_{\text {exp }}$. However, for $\left\langle{ }^{11} \mathrm{~B} \mid{ }^{10} \mathrm{~B}\right\rangle$ and $\left\langle{ }^{11} \mathrm{C} \mid{ }^{10} \mathrm{~B}\right\rangle$ agreement is observed only for the second $3 / 2^{-}$state in ${ }^{11} \mathrm{C}$. This indicates that the experimental ANCs for $A=11$ are most likely overestimated.

\section{F. $A=\mathbf{1 0}$}

The ANC for the overlap $\left\langle{ }^{10} \mathrm{~B} \mid{ }^{9} \mathrm{Be}\right\rangle$ has been obtained by using two different peripheral transfer reactions, ${ }^{9} \mathrm{Be}\left({ }^{3} \mathrm{He}, d\right){ }^{10} \mathrm{~B}$ [34] and ${ }^{9} \mathrm{Be}\left({ }^{10} \mathrm{~B},{ }^{9} \mathrm{Be}\right){ }^{10} \mathrm{~B}$ [59]. For the ${ }^{10} \mathrm{~B}$ ground state, the ANCs obtained with these two reactions agree with each other. However, for excited states in ${ }^{10} \mathrm{~B}$, the two available experimental determinations result in different ANCs.

The theoretical predictions for the ${ }^{10} \mathrm{~B}$ ground-state $\mathrm{ANC}$ are not very sensitive to the shell-model interaction, so this ANC is determined mostly by $\tilde{V}^{\text {eff }}$. This theoretical ANC is significantly smaller than $C_{\text {exp }}$. The mirror neutron ANC is in reasonable agreement with the one obtained via the ${ }^{10} \mathrm{~B}(d, t)^{9} \mathrm{~B}$ reaction; however, it does not satisfy the mirror symmetry relations as $\mathcal{R}_{\text {exp }}=2.75(68)$, calculated with experimental ANCs from mirror $\left(d,{ }^{3} \mathrm{He}\right)$ and $(d, t)$ reactions, is much larger than the value of 1.21 obtained from the analytical formula from Ref. [37]. Such a disagreement is not expected as the proton and neutron separation energies involved are large and the SFs for this overlap are large as well.

For all other excited states, the two experimental ANCs determined via the $\left({ }^{3} \mathrm{He}, d\right)$ and $\left({ }^{10} \mathrm{~B},{ }^{9} \mathrm{Be}\right)$ reactions are different. Apart from the second $1^{+}$state where the theoretical ANC agrees with the $\left({ }^{10} \mathrm{~B},{ }^{9} \mathrm{Be}\right)$ value, the theoretical ANCs for two other ${ }^{10} \mathrm{~B}$ states lie between the two available experimental values. The SFs $S^{\mathrm{IE}}$ are reduced from $S^{\mathrm{DE}}$ for all the cases considered.

\section{G. $A=9$}

The overlap $\left\langle\left.{ }^{9} \mathrm{C}\right|^{8} \mathrm{~B}\right\rangle$ is important in connection with the explosive hydrogen burning reaction ${ }^{8} \mathrm{~B}(p, \gamma){ }^{9} \mathrm{C}$. The three experimental studies for this overlap that are available [61-63] give overlapping results for its ANC. The sensitivity of theoretical ANC to the shell-model potential is about $20 \%$. The Millener interaction gives the ${ }^{9} \mathrm{C}$ ANC, which agrees with $C_{\text {exp }}$ from two experimental studies, and the $\mathrm{SF} S^{\mathrm{IE}}$ that is just on the low edge of the $S_{\text {exp }}$ range determined by the error bars. For the mirror overlap, $\left\langle{ }^{9} \mathrm{Li} \mid{ }^{8} \mathrm{Li}\right\rangle$, an agreement exists between the experimental and theoretical ANCs as well and the experimental ${ }^{9} \mathrm{Li}$ and ${ }^{9} \mathrm{C}$ ANCs satisfy the mirror-symmetry relation. The SFs $S^{\mathrm{IE}}$ are reduced with respect to the $S^{\mathrm{DE}}$. For ${ }^{9} \mathrm{Li}$, they agree with $S_{\text {exp }}$ obtained in the ${ }^{9} \mathrm{Li}(d, t){ }^{8} \mathrm{Li}$ reaction where the overlap calculated in the VMC calculations has been used in the analysis.

For another overlap, $\left\langle{ }^{9} \mathrm{Be} \mid{ }^{8} \mathrm{Be}\right\rangle$, where the experimental data are available, the calculations give ANCs that are significantly smaller than $C_{\text {exp }}$ for all three shell-model interactions. The SFs $S^{\mathrm{IE}}$ are reduced with respect to the $S^{\mathrm{DE}}$.

\section{H. $A=8$}

For the weakly bound $A=8$ isotopes, ${ }^{8} \mathrm{Li}$ and ${ }^{8} \mathrm{~B}$, experimental ANC values are available. They have been studied in Refs. [64-66] in connection with the solar $p p$ chain reaction ${ }^{7} \mathrm{Be}(p, \gamma)^{8} \mathrm{~B}$. In particular, the ratio $\left(C_{p 1 / 2} / C_{p 3 / 2}\right)^{2}=$ $0.13(2)$ has been determined in ${ }^{8} \mathrm{Li} \mathrm{[64]}$. This ratio is very sensitive to the shell-model potential employed to calculate the nuclear wave functions. None of the potentials used here has reproduced this ratio, which is $0.157,0.034$, and 0.093 for Boyarkina, CK816, and Millener interactions, respectively. The first of these numbers was used in Refs. [65,66] to obtain the ${ }^{8} \mathrm{~B}$ ANC. As it is higher than the ratio of $0.13(2)$ determined for the ${ }^{8} \mathrm{Li}$ independently, some additional uncertainty should be present in $C_{\text {exp }}$ for ${ }^{8} \mathrm{~B}$. As for the ANCs themselves, the best agreement with the experimental results is obtained if the Millener interaction is used; however, they are still slightly lower than the experimental ones.

For strongly bound nucleons in $A=8$ nuclei, $S^{\mathrm{IE}}$ is approximately half the value of $S^{\mathrm{DE}}$. The reduction factor $R_{S}$ is $0.42-0.5$ for $\left\langle{ }^{8} \mathrm{Li} \mid{ }^{7} \mathrm{He}\right\rangle$ whereas for $\left\langle{ }^{8} \mathrm{Be} \mid{ }^{7} \mathrm{Li}\right\rangle$ it is even smaller, $R_{s}=0.39 . S^{\mathrm{IE}}$ for $\left\langle\left.{ }^{8} \mathrm{Li}\right|^{7} \mathrm{He}\left(3 / 2^{-}\right)\right\rangle$agrees with $S_{\text {exp }}$ obtained in Ref. [68] using the form factor for the transferred proton consistent with the VMC calculations but for $\left\langle\left.{ }^{8} \mathrm{Li}\right|^{7} \mathrm{He}\left(5 / 2^{-}\right)\right\rangle$, $S^{\mathrm{IE}}$ is slightly smaller than $S_{\text {exp }}$.

For the weakly bound proton in ${ }^{8} \mathrm{~B}, S^{\mathrm{IE}}$ is reduced from $S^{\mathrm{DE}}$ to a much lesser extent, the corresponding reduction factor of $0.67-0.86$ being higher than that from protons in ${ }^{8} \mathrm{Li}$ and ${ }^{8} \mathrm{Be}$. This SF has been studied in the proton knockout reaction [63]. $S_{\text {exp }}=0.89$ (7) can be deduced from this work by assuming that the cross-section reduction observed in this reaction is due to the reduction of the shell-model SF. The present value of 0.783 is slightly below this value.

\section{I. $A=7$}

For $\left\langle{ }^{7} \mathrm{Li} \mid{ }^{6} \mathrm{Li}\right\rangle$, the ANCs are available from the analysis of the $(d, t)$ transfer reaction [51]. The theoretical predictions for ANCs made with different shell-model potentials are similar, excluding the $j=3 / 2$ component in the final ground state, ${ }^{6} \mathrm{Li}\left(1^{+}\right)$, where CK816 gives a significantly smaller value. This occurs owing to a larger component $\left|[42]^{13} \mathrm{D}\right\rangle$ in ${ }^{6} \mathrm{Li}\left(1^{+}\right)$ predicted by CK816. The ANCs for the $\left\langle{ }^{7} \mathrm{Li} \mid{ }^{6} \mathrm{Li}\left(1^{+}\right)\right\rangle$and $\left\langle{ }^{7} \mathrm{Li} \mid{ }^{6} \mathrm{Li}\left(3^{+}\right)\right\rangle$overlaps are in good agreement with experimental ones. However, the ANC for the final state ${ }^{6} \mathrm{Li}\left(0^{+}\right)$, which is a mirror analog of the ground state of ${ }^{6} \mathrm{He}$, is smaller than the experimental one. This is consistent with the underestimation of the neutron SF obtained in the $d\left({ }^{7} \mathrm{Li}, t\right)^{6} \mathrm{Li}$ reaction in 
Ref. [68] and the proton SF obtained from ${ }^{7} \mathrm{Li}\left(e, e^{\prime} p\right)^{6} \mathrm{He}$ and $d\left({ }^{7} \mathrm{Li},{ }^{3} \mathrm{He}\right){ }^{6} \mathrm{Li}$ reactions (see Table VII). All the SFs $S^{\mathrm{IE}}$ for ${ }^{7} \mathrm{Li}$ are reduced by approximately a factor of 2 with respect to $S^{\mathrm{DE}}$.

\section{ENERGY DEPENDENCE OF ANCS AND SPECTROSCOPIC FACTORS}

A comparison of the theoretical and experimental ANCs in the previous section shows that the choice of the Millener interaction to generate the weights of the shell-model configurations combined with M3YE for the $\tilde{V}^{\text {eff }}$ effective potential is on average very reasonable. With this combination, the ratio $C_{\mathrm{exp}}^{2} / C_{\mathrm{th}}^{2}$, plotted on Fig. 6 for the ground states of the $0 p$-shell nuclei as a function of $\Delta S$, are concentrated around one, excluding a few points in the middle of the $0 p$ shell. The average deviation from one in either direction is about $25 \%$. For other choices of $\tilde{V}^{\text {eff }}$, such as M3YR or M3YP, $C_{\text {exp }}^{2} / C_{\text {th }}^{2}$ would be concentrated around a larger value. No energy dependence is seen in this plot, which suggests that the source term is calculated fairly well for most of the $p$-shell nuclei. Therefore, the same source term can be used to study the energy (or $\Delta S$ ) dependence of SFs.

The ratio $R_{\mathrm{DE}}^{\mathrm{IE}}=S^{\mathrm{IE}} / S^{\mathrm{DE}}(\Delta S)$ is plotted for ground states of $0 p$-shell nuclei in Fig. 7 for four different situations: (i) when the angular momentum $j=1 / 2$ is removed from the nucleus, which in a simple independent-particle model would be considered as a $p_{1 / 2}$ nucleus (red squares); (ii) when $j=3 / 2$ is removed from the $p_{3 / 2}$ nuclei (black circles); (iii) when $j=3 / 2$ is removed from the $p_{1 / 2}$ nuclei (orange diamonds), and (iv) when $j=1 / 2$ is removed from the $p_{3 / 2}$ nuclei (blue triangles). In all four cases $R_{\mathrm{DE}}^{\mathrm{IE}}$ decreases toward large positive $\Delta S$. It is also noticeable that the $\Delta S$ dependence for cases (i) and (ii) is similar but the corresponding regions of $R_{\mathrm{DE}}^{\mathrm{IE}}$ are split from each other. They are noticeably flatter than the $R_{S}(\Delta S)$ obtained in Ref. [5] from the knockout reactions. Cases (iii) and (iv) correspond to small

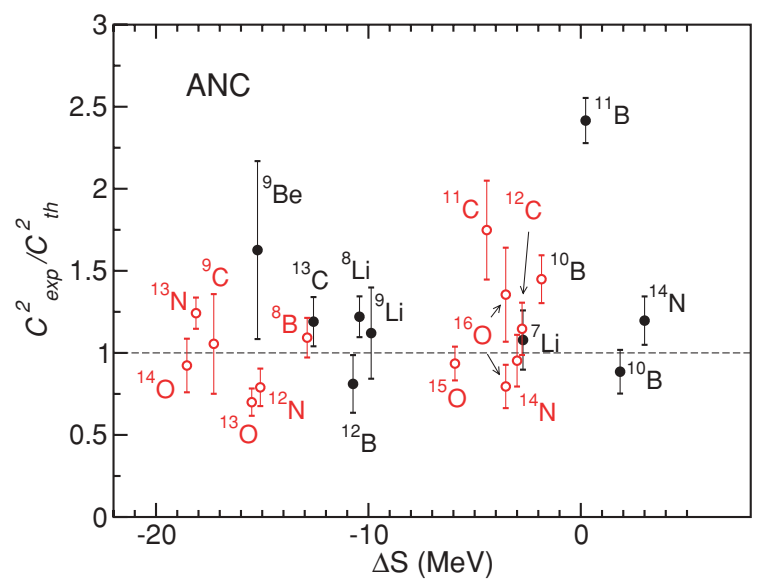

FIG. 6. (Color online) The ratio of experimental to theoretical ANCs squared for neutrons (black filled circles) and protons (red open circles), calculated with the Millener wave functions and the M3YE potential, for a range of the ground state of $0 p$-shell nuclei as a function of $\Delta S$ taken as $S_{p}-S_{n}$ or $S_{n}-S_{p}$ for proton or neutron removal, respectively.

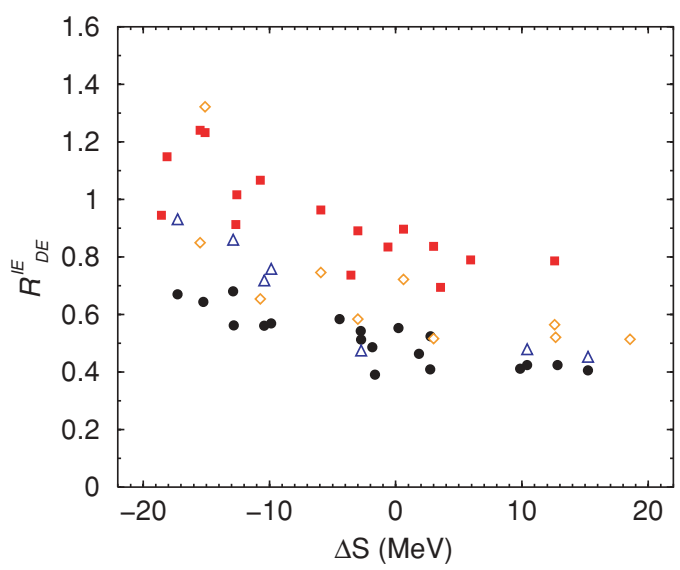

FIG. 7. (Color online) The ratio $R_{\mathrm{DE}}^{\mathrm{IE}}(\Delta S)$ calculated with the Millener wave functions and the M3YE potential for four different situations as explained in the text. $\Delta S$ is $S_{p}-S_{n}$ or $S_{n}-S_{p}$ for proton or neutron removal, respectively.

SFs and they have stronger $\Delta S$ dependence. When $R_{\mathrm{DE}}^{\mathrm{IE}}(\Delta S)$ is calculated as $\left(S_{j=1 / 2}^{\mathrm{IE}}+S_{j=3 / 2}^{\mathrm{IE}}\right) /\left(S_{j=1 / 2}^{\mathrm{DE}}+S_{j=3 / 2}^{\mathrm{DE}}\right)$ it has a similar $\Delta S$ dependence. In the previous publication [11], some components of the M3YE were renormalized, which improved the agreement between the theoretical and experimental ANCs and moved the $R_{\mathrm{DE}}^{\mathrm{IE}}(j=3 / 2)$ and $R_{\mathrm{DE}}^{\mathrm{IE}}(j=1 / 2)$ toward each other, thus reducing the thickness of $R_{\mathrm{DE}}^{\mathrm{IE}}$. However, a closer examination has shown that the renormalized M3YE gives a dramatic increase of the SF for the overlap between ${ }^{16} \mathrm{O}$ and the ${ }^{15} \mathrm{~N}\left(3 / 2^{-}\right)$excited state. Because of that, no renormalization of $\tilde{V}^{\text {eff }}$ is made in the present paper. There are other factors that have not been accounted for, which could reduce the thickness and/or change the slope of $R_{\mathrm{DE}}^{\mathrm{IE}}(\Delta S)$.

The $\Delta S$ behavior of $R_{\mathrm{DE}}^{\mathrm{IE}}$ originates from the energy dependence (via $\kappa$ ) of the Green's function in Eq. (2). Figure 8 shows a few examples of $j=1 / 2$ overlaps calculated with a fixed source term as a function of the separation energy $\varepsilon$ of the removed nucleon. One can see that $R_{\mathrm{DE}}^{\mathrm{IE}}$ increases as $\varepsilon$ decreases. Thus, the SFs in weaker bound nuclei should

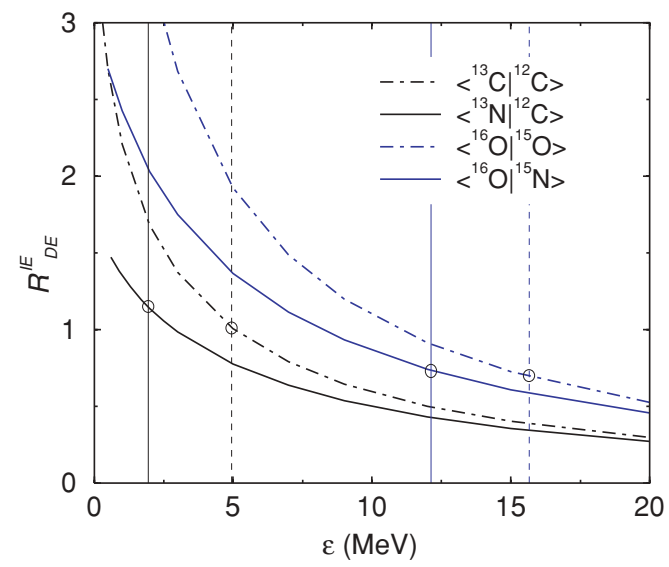

FIG. 8. (Color online) The ratio $R_{\mathrm{DE}}^{\mathrm{IE}}$ as a function of separation energy $\varepsilon$ of the removed nucleon calculated with the Millener wave functions and the M3YE potential for four overlap integrals. Vertical lines indicate experimental binding energies. 
be less reduced with respect to the shell-model values than those in more strongly bound nuclei. This contradicts the prediction of the Gamow shell model, which states that because of the coupling to the continuum the SFs in weakly bound nuclei should be more strongly reduced from the shell-model ones [70]. The Gamow shell model also predicts the existence of cusps in the energy behavior of SFs near the nucleon emission threshold $\varepsilon=0$. No near-threshold cusps are seen in the present calculations. This could be a consequence of the absence of any energy dependence in the shell-model source term. At smaller energies the radius of the source term should increase, which should lead to smaller SFs near $\varepsilon=0$.

\section{COMPARISON TO VMC CALCULATIONS}

For some $A \leqslant 10$ nuclei, the $a b$ initio VMC calculations for one-nucleon overlap functions are available [69]. The corresponding SFs $S_{a b}$ are shown in Table VII. These SFs are systematically smaller than the shell-model values $S_{l j}^{\mathrm{DE}}$ for strongly bound nuclei while for ${ }^{8} \mathrm{Li}$ and ${ }^{9} \mathrm{Li}$ (the mirror analogs of weakly bound nuclei ${ }^{8} \mathrm{~B}$ and ${ }^{9} \mathrm{C}$ ) they are close to $S_{l j}^{\mathrm{DE}}$. This confirm the $R_{s}(\Delta S)$ dependence observed in knockout reactions. However, except for three cases, $\left\langle{ }^{7} \mathrm{Li} \mid{ }^{6} \mathrm{He}\left(0^{+}\right)\right\rangle,\left\langle{ }^{7} \mathrm{Li} \mid{ }^{6} \mathrm{Li}\left(1^{+}\right)\right\rangle$, and $\left\langle{ }^{8} \mathrm{Li} \mid{ }^{7} \mathrm{He}\left(5 / 2^{-}\right)\right\rangle, S_{a b}$ values are larger than those of $S_{\text {exp }}$. However, the SFs $S_{l j}^{\mathrm{IE}}$ obtained in the present approach are closer to $S_{\text {exp }}$, except for $\left\langle{ }^{7} \mathrm{Li} \mid{ }^{6} \mathrm{He}\left(0^{+}\right)\right\rangle$.

A more detailed comparison between the overlaps $I_{l j}^{\mathrm{IE}}(r)$ generated by the shell-model source term and the overlaps $I_{l j}^{a b}(r)$ obtained in VMC calculations is presented in Fig. 9. Also shown are the overlaps $I_{l j}^{\mathrm{IE}}(r)$ multiplied by a renormalization factor to match $I_{l j}^{a b}(r)$ at the maximum to give an idea about differences in their shapes. Figure 9 reveals that for $j=1 / 2$, the shapes of $I_{l j}^{\mathrm{IE}}(r)$ and $I_{l j}^{a b}(r)$ are very similar.
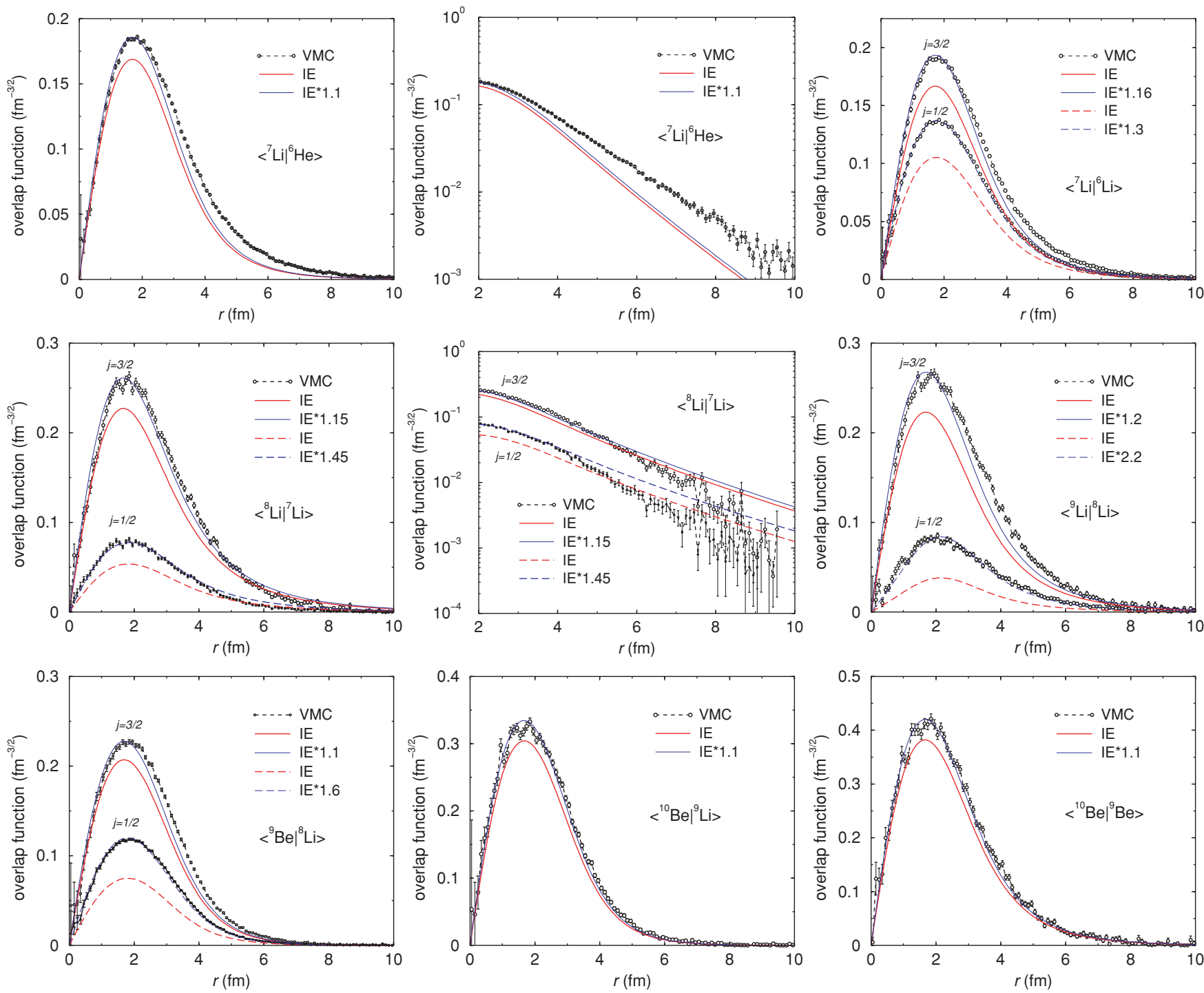

FIG. 9. (Color online) Absolute values for the overlap integrals calculated with M3YE and the Millener interaction as the solution of the inhomogeneous equation in comparison to those obtained in ab initio VMC calculations. Also shown are the overlaps from the inhomogeneous equation renormalized to match the ab initio result at maximum. The overlaps $\left\langle{ }^{7} \mathrm{Li} \mid{ }^{6} \mathrm{He}\right\rangle$ and $\left\langle{ }^{8} \mathrm{Li} \mid{ }^{7} \mathrm{Li}\right\rangle$ are also shown on a logarithmic scale. 
However, for $j=3 / 2$ two situations occur. For lighter nuclei, ${ }^{7,8,9} \mathrm{Li}$ and ${ }^{9} \mathrm{Be}, I_{l j}^{\mathrm{IE}}(r)$ has a significantly smaller radius than $I_{l j}^{a b}(r)$. This may originate in the cluster structure of the Li and Be isotopes. In particular, removing one proton from ${ }^{7} \mathrm{Li}$ leads to a Borromean halo nucleus ${ }^{6} \mathrm{He}$, where two neutrons stay far away from the ${ }^{4} \mathrm{He}$ core. Even if the removed proton is far away from the center of mass of ${ }^{7} \mathrm{Li}$, it can still be close to one of the halo neutrons interacting with it, which results in abnormal preasymptotic behavior of $I_{l j}^{a b}(r)$. Indeed, this overlap can be reproduced by a two-body wave function calculated in the potential model with the diffuseness of $1.25 \mathrm{fm}$. With such a huge diffuseness, the Coulomb barrier is dramatically reduced and the true asymptotic behavior of the overlap is achieved only after $r=12 \mathrm{fm}$. Abnormal preasymptotic behavior has been predicted in Ref. [71] and has been investigated later in Ref. [72] for several three-body systems. It was shown there that strong interaction between the removed nucleon and the weakly bound valence nucleons from the residue leads to increased rms radii of overlap functions as compared to predictions made with standard Woods-Saxon geometry. The preasymptotic abnormalities should influence the source term as well; however, the oscillator shell model used here for its calculations cannot reproduce this effect. It is interesting that, for a heavier nucleus, ${ }^{10} \mathrm{Be}$, the shapes of $I_{l j=3 / 2}^{\mathrm{IE}}(r)$ and $I_{l j=3 / 2}^{a b}(r)$ are similar. This may mean that mean-field components of the wave functions become more important than the cluster effects with increasing $A$.

In the present approach, the rms radius of $I_{l j=3 / 2}^{\mathrm{IE}}(r)$ is always smaller than that for $I_{l j=1 / 2}^{\mathrm{IE}}(r)$ for the same nucleus. Similar predictions are made by VMC but the difference between the rms radii of $I_{l j=3 / 2}^{a b}(r)$ and $I_{l j=1 / 2}^{a b}(r)$ is smaller. This can be seen by plotting both overlaps on the same graph and renormalizing them to match at maximum (not shown here). The ratio $S_{l j=1 / 2}^{\mathrm{IE}} / S_{l j=3 / 2}^{\mathrm{IE}}$ is always smaller than $S_{l j=1 / 2}^{a b} / S_{l j=3 / 2}^{a b}$ predicted by VMC. In particular, for ${ }^{8} \mathrm{Li}$ the VMC ratio is 0.22 . This is much larger than $S_{l j=1 / 2}^{\mathrm{IE}} / S_{l j=3 / 2}^{\mathrm{IE}}=0.076$ obtained with the Millener interaction. It is also much larger than the ANC ratio squared, $C_{l j=1 / 2}^{2} / C_{l j=3 / 2}^{2}=0.13(2)$, deduced from the neutron transfer experiment.

While VMC is expected to give a good result for the overlap functions inside the nuclear interior, it cannot guarantee the correct behavior at large $r$. To illustrate this, two overlaps are shown in Fig. 9 on a logarithmic scale as well. In the first case, $\left\langle{ }^{7} \mathrm{Li} \mid{ }^{6} \mathrm{He}\left(0^{+}\right)\right\rangle$, the $I_{l j}^{a b}(r)$ overlap decreases significantly slower than required by the experimental proton separation energy of $9.975 \mathrm{MeV}$. The VMC does not reproduce this energy, with the difference between calculated ${ }^{7} \mathrm{Li}$ and ${ }^{6} \mathrm{He}$ energies being $8.31 \mathrm{MeV}$. The $I_{l j}^{a b}(r)$ behavior at large $r$ is indeed consistent with $\varepsilon=8.31 \mathrm{MeV}$ within statistical uncertainties. In contrast, $I_{l j}^{\mathrm{IE}}(r)$ has the correct decrease shown in the same graph. In the second case, $\left\langle{ }^{8} \mathrm{Li} \mid{ }^{7} \mathrm{Li}\right\rangle$, the difference $E\left({ }^{8} \mathrm{Li}\right)-E\left({ }^{7} \mathrm{Li}\right)$ is $2.6 \mathrm{MeV}$, so that, according to $\mathrm{VMC},{ }^{8} \mathrm{Li}$ should be unbound and, therefore, this overlap should have an oscillating asymptotic behavior. Nevertheless, the VMC overlap $I_{l j}^{a b}(r)$ shows a decrease typical for a bound nucleus with $\varepsilon>2.03 \mathrm{MeV}$, which is the experimental value for the neutron separation energy in ${ }^{8} \mathrm{Li}$. A possible way to achieve correct asymptotic behavior within the VMC is to use it for the source term calculations.

\section{CONCLUSIONS}

The procedure of direct evaluation of overlap integrals between the model wave functions of two neighboring nuclei, widely used for the calculations of one-nucleon overlap functions and SFs, is sensitive only to effective interactions in the truncated model space. However, the inhomogeneous equation tells us that the overlap functions and the corresponding SFs must depend on contributions from the excluded model space. Therefore, important information about nuclear structure is lost when overlaps and the SFs are calculated in a standard shell model. The contributions from the excluded space can be routinely recovered by calculating the source term and supplying it to the inhomogeneous equation even if the simplest model spaces are used for these purposes, provided a proper choice of the effective $N N$ interaction is made. As a bonus, this method guarantees the correct asymptotic decrease for the overlap so that the ANCs needed for many applications, including astrophysical ones, can be obtained.

A simple modeling of the effective interaction by an $A$-independent two-body local interaction provides the ANCs for the ground states of $p$-shell nuclei that are in fair agreement with experimental ones, deviating on average by $\sim 25 \%$. Further tuning of this potential may improve agreement between the experimental and theoretical ANCs. However, it would be more beneficial to try to understand this potential from first principles. In particular, it is very important to clarify whether this potential is state dependent or nonlocal and what the role of the three-body or higher order contributions is.

The SFs obtained from overlap functions generated by the shell-model source term show deviation from original shellmodel values. This deviation depends on separation energies and, when plotted as a function of $\Delta S$, shows the same trend as $R_{S}(\Delta S)$ observed in one-nucleon knockout reactions. This energy dependence is inherited, at least partially, from the $\kappa$ dependence of the Green's function. Thus, the inhomogeneous equation offers a new perspective for studying the SF reduction phenomenon. Extending the standard shell model and more advanced microscopic techniques, including ab initio methods, for these purposes would be highly beneficial.

\section{ACKNOWLEDGMENTS}

I am grateful to D.J. Millener who has for provided me with his supermultiplet shell-model code, the $N N$ interactions, and some missing coefficients of fractional parentage. He has kindly helped me with running the code and engaged in valuable discussions. I am also grateful to M. Viviani and A. Kievsky for sending me overlap functions of three- and four-body systems and to R. Wiringa for providing the updated values of the VMC binding energies. The support from UK STFC Grant No. ST/F012012/1 is also acknowledged. 


\section{APPENDIX}

To calculate the source term the fractional parentage expansion technique has been used. The wave functions of the $A-1$ and $A$ nuclei have been expanded over the complete set of translationally invariant shell-model states of the $A-2$ nucleus. To evaluate the arising integrals the Fourier transform of the effective $N N$ interaction $\tilde{V}^{\text {eff }}$ has been used. The final analytical expressions for the case of vertex form factors are given in Ref. [21]. Only minor modifications are required to get the source term.

Important inputs to the calculations of the source term are one-body and two-body coefficients of fractional parentage (CFPs) of the translationally invariant shell model. The general analytical expressions for these coefficients are given for $0 p$-shell nuclei in Ref. [73]. The analytical expressions for CFPs specifically required for the source term calculations are given in this appendix.

\section{A. One-body CFPs}

The one-body fractional parentage expansion reads

$$
\begin{aligned}
\left|A N \gamma M_{L} M_{S} M_{T}\right\rangle & \sum_{\substack{N^{\prime} \gamma^{\prime} n l \sigma \tau \\
M_{L}^{\prime} M_{S}^{\prime} M_{T}^{\prime}}}\left\langle A N \gamma \mid A-1 N^{\prime} \gamma^{\prime}, n l\right\rangle\left(L^{\prime} M_{L}^{\prime} \operatorname{lm} \mid L M_{L}\right) \\
& \times\left(S^{\prime} M_{S}^{\prime} \frac{1}{2} \sigma \mid S M_{S}\right)\left(T^{\prime} M_{T}^{\prime} \frac{1}{2} \tau \mid T M_{T}\right) \\
& \times\left|A-1 N^{\prime} \gamma^{\prime} M_{L}^{\prime} M_{S}^{\prime} M_{T}^{\prime}\right\rangle \varphi_{n l m}\left(\xi_{A-1}\right) \chi_{\sigma \tau}^{S T}(A),
\end{aligned}
$$

where $\varphi_{n l m}$ is the oscillator wave function of nucleon $A$ that depends on normalized Jacobi coordinate $\boldsymbol{\xi}_{A-1}, \chi_{\sigma \tau}^{S T}(A)$ is the spin-isospin wave function of this nucleon, and $\left\langle A N \gamma \mid A-1 N^{\prime} \gamma^{\prime}, n l\right\rangle$ is the one-body CFP. The meaning of all the quantum numbers is explained in Sec. V. For $0 p$-shell nuclei and the minimal possible number of oscillator quanta, $N$, two values of $N^{\prime}$ are allowed, $N^{\prime}=N-1$ and $N^{\prime}=N$. According to Ref. [73], when one nucleon is removed from the $0 p$ shell then

$$
\begin{aligned}
& \left\langle A N \gamma \mid A-1 N^{\prime}=N-1 \gamma^{\prime}, 0 p\right\rangle \\
& =-\left(\frac{A-4}{A-1}\right)^{1 / 2}\left(\frac{n_{f_{p}^{\prime}}}{n_{f_{p}}}\right)^{1 / 2}\left\langle p^{A-4}\left[f_{p}\right] \beta L\right| p^{A-5}\left[f_{p}^{\prime}\right] \\
& \left.\quad \times \beta^{\prime} L^{\prime}, 0 p\right\rangle\left\langle(s t)^{A}[\tilde{f}] S T \mid(s t)^{A-1}\left[\tilde{f}^{\prime}\right] S^{\prime} T^{\prime}, \frac{1}{2} \frac{1}{2}\right\rangle,
\end{aligned}
$$

where $\left[f_{p}\right]$ is the irreducible representation (irrep) of the permutation group for the $A-4$ nucleons of the $0 p$ shell obtained from $[f]$ by eliminating the upper row, $n_{f_{p}}$ is the dimension of this irrep, $\left\langle p^{A-4}\left[f_{p}\right] \beta L \mid p^{A-5}\left[f_{p}^{\prime}\right] \beta^{\prime} L^{\prime}, 0 p\right\rangle$ is the one-body orbital CFP given by the Clebsch-Gordan coefficients for the $\mathrm{SU}(3)$ group [74], and the $\left\langle(s t)^{A}[\tilde{f}] S T \mid(s t)^{A-1}\left[\tilde{f}^{\prime}\right] S^{\prime} T^{\prime}, \frac{1}{2} \frac{1}{2}\right\rangle$ is the one-body spin-isospin CFP given by the Clebsch-Gordan coefficients for the SU(4) group [75]. When one nucleon is removed from the $0 s$ shell then

$$
\begin{aligned}
& \left\langle A N \gamma \mid A-1 N^{\prime}=N \gamma^{\prime}, 0 s\right\rangle \\
& =(-)^{A}\left(\frac{4}{A}\right)^{1 / 2} \Omega_{N^{\prime} \gamma^{\prime}}(A-1)\left(\begin{array}{lll}
{[\tilde{3}]} & {[\tilde{1}]} & {[\tilde{4}]} \\
{\left[\tilde{f}_{p}^{\prime}\right]} & 0 & {[\tilde{f}]} \\
{\left[\tilde{f}^{\prime}\right]} & {[\tilde{1}]} & {[\tilde{f}]}
\end{array}\right) \\
& \quad \times\left\langle(s t)^{A}[\tilde{f}] S T \mid(s t)^{A-1}\left[\tilde{f}^{\prime}\right] S^{\prime} T^{\prime}, \frac{1}{2} \frac{1}{2}\right\rangle .
\end{aligned}
$$

The coefficient $\Omega_{N \gamma}$ for this particular case is found from [73]

$$
\Omega_{N \gamma}^{2}(A)=1-\frac{A-3}{A} \frac{N\left(\left[\tilde{f}_{p}\right]\right) n\left(\left[f_{p_{1}}\right]\right)}{n\left(\left[f_{p}\right]\right) N\left(\left[\tilde{f}_{p_{1}}\right]\right)},
$$

where the Young diagram $\left[f_{p_{1}}\right]$ is determined by $(\lambda \mu)$ from $\gamma,[\tilde{f}]$ denotes the conjugate Young diagram, and $N[\tilde{f}]$ is the dimension of the irrep $[\tilde{f}]$ for the group $\mathrm{SU}(4)$. The remaining symbol in Eq. (A3) in round brackets is the $9 j$ symbol for the SU(4) group [76].

\section{B. Two-body CFPs}

The two-body fractional parentage expansion reads

$$
\begin{aligned}
\mid A N & \left.\gamma M_{L} M_{S} M_{T}\right\rangle \\
= & \sum_{\substack{N^{\prime} \gamma^{\prime} n l N L_{0} \\
L_{2} S_{2} T_{2}\{\mu\}}}\left|A-2 N^{\prime} \gamma^{\prime} M_{L}^{\prime} M_{S}^{\prime} M_{T}^{\prime}\right\rangle \\
& \times\left\langle A N \gamma \mid A-2 N^{\prime} \gamma^{\prime}, N L_{0}, n l S T\left(L_{2}\right)\right\rangle\left(L_{0} M_{0} l m \mid L_{2} M_{2}\right) \\
& \times\left(L^{\prime} M_{L}^{\prime} L_{2} M_{2} \mid L M_{L}\right) \varphi_{n l m}\left(\xi_{A-1, A}\right) \varphi_{N L_{0} M_{0}}\left(\xi_{2}\right) \\
& \times\left(S^{\prime} M_{S}^{\prime} S_{2} M_{S_{2}} \mid S M_{S}\right)\left(T^{\prime} M_{T}^{\prime} T_{2} M_{T_{2}} \mid T M_{T}\right) \\
& \times \chi_{S_{2} M_{S_{2}} T_{2} M_{T_{2}}}^{S T}(A-1, A),
\end{aligned}
$$

where $\quad\{\mu\}=\left\{m M_{0} M_{2} M_{L}^{\prime} M_{S}^{\prime} M_{T}^{\prime} M_{S_{2}} M_{T_{2}}\right\}, \quad \varphi_{N L_{0} M_{0}} \quad$ and $\chi_{S_{2} M_{S_{2}} T_{2} M_{T_{2}}}^{S T}(A-1, A)$ are the orbital and the spin-isospin wave functions of the removed pair $(A-1, A)$, respectively, and $\varphi_{n l m}$ is the wave function of the relative motion between the removed pair of nucleons and the center of mass of $A-2 . \xi_{2}$ and $\boldsymbol{\xi}_{A-1, A}$ are the corresponding normalized Jacobi coordinates. $\left\langle A N \gamma \mid A-2 N^{\prime} \gamma^{\prime}, N L_{0}, n l S T\left(L_{2}\right)\right\rangle$ is the two-body CFP that is required for two values of $N^{\prime}, N=$ $N-2$ and $N^{\prime}=N-1$. In the first case, according to Ref. [73],

$$
\begin{aligned}
&\langle A N \gamma\left|A-2 N^{\prime}=N-2 \gamma^{\prime}, N L_{0}, n l S T\left(L_{2}\right)\right\rangle \\
&=(-)^{A-5+N}\left(\frac{16(A-4)}{A(A-1)}\right)^{1 / 2}\left(\frac{n_{f_{p}^{\prime}}}{n_{f_{p}}}\right)^{1 / 2}\left(\frac{A}{A-2}\right)^{N / 2} \\
& \quad \times\left\langle p^{A-4}\left[f_{p}\right] \beta L \mid p^{A-6}\left[f_{p}^{\prime}\right] \beta^{\prime} L^{\prime}, L_{2}\right\rangle \\
& \quad \times\left\langle 0 p 0 p: L_{2} \mid N L_{0} n l: L_{2}\right\rangle \\
& \quad \times\left\langle(s t)^{A}[\tilde{f}] S T \mid(s t)^{A-2}\left[\tilde{f}^{\prime}\right] S^{\prime} T^{\prime}, S_{2} T_{2}\right\rangle
\end{aligned}
$$

where $\left\langle p^{A-4}\left[f_{p}\right] \beta L \mid p^{A-6}\left[f_{p}^{\prime}\right] \beta^{\prime} L^{\prime}, L_{2}\right\rangle$ is the two-body orbital CFP, $\left\langle(s t)^{A}[\tilde{f}] S T \mid(s t)^{A-2}\left[\tilde{f}^{\prime}\right] S^{\prime} T^{\prime}, S_{2} T_{2}\right\rangle$ is the twobody spin-isospin CFP, and $\left\langle 0 p 0 p: L_{2} \mid N L_{0} n l: L_{2}\right\rangle$ is the 
Talmi-Moshinsky transformation coefficient for particles with equal masses [77]. In the second case,

$$
\begin{aligned}
& \left\langle A N \gamma \mid A-2 N^{\prime}=N-1 \gamma^{\prime}, N L_{0}, n l S T\left(L_{2}\right)\right\rangle \\
& =(-)^{A-5+N}\left(\frac{16(A-4)}{A(A-1)}\right)^{1 / 2}\left(\frac{n_{f_{p}^{\prime}}}{n_{f_{p}}}\right)^{1 / 2}\left(\frac{A}{A-2}\right)^{N / 2} \\
& \quad \times\left\langle p^{A-4}\left[f_{p}\right] \beta L \mid p^{A-5}\left[f_{p}^{\prime}\right] \beta^{\prime} L^{\prime}, 0 p\right\rangle\left\langle 0 s 0 p: 1 \mid N L_{0} n l: 1\right\rangle \\
& \quad \times \Omega_{N^{\prime} \gamma^{\prime}}(A-2)\left(\begin{array}{lll}
{[\tilde{3}]} & {[\tilde{1}]} & {[\tilde{4}]} \\
{\left[\tilde{f}_{p}^{\prime}\right]} & {[\tilde{1}]} & {\left[\tilde{f}_{p}\right]} \\
{\left[\tilde{f}^{\prime}\right]} & {\left[\tilde{f}_{2}\right]} & {[\tilde{f}]}
\end{array}\right) \\
& \quad \times\left\langle(s t)^{A}[\tilde{f}] S T \mid(s t)^{A-2}\left[\tilde{f}^{\prime}\right] S^{\prime} T^{\prime}, S_{2} T_{2}\right\rangle .
\end{aligned}
$$

Here the $\left[f_{2}\right]$ is the Young diagram determined by $\left\{l S_{2} T_{2}\right\}$. As in the previous section, $\left[f_{p}\right]$ and $\left[f_{p}^{\prime}\right]$ are determined by the Elliott symbols $(\lambda \mu)$ and $\left(\lambda^{\prime} \mu^{\prime}\right)$, respectively.

\section{C. $9 j$ symbols for the $\mathrm{SU}(4)$ group}

The main difficulty in calculating the CFPs that contain the $A-2$ states with $N^{\prime} \neq N_{\text {min }}$ is the evaluation of the $9 j$ symbols for the $\mathrm{SU}(4)$ group. Fortunately, to calculate the source term only the product of two $9 j$ coefficients is required,

$$
\left(\begin{array}{lll}
{[\tilde{3}]} & {[\tilde{1}]} & {[\tilde{4}]} \\
{\left[\tilde{f}_{p}^{\prime}\right]} & 0 & {\left[\tilde{f}_{p}^{\prime}\right]} \\
{\left[\tilde{f}^{\prime}\right]} & {[\tilde{1}]} & {\left[\tilde{f}_{A-1}\right]}
\end{array}\right)\left(\begin{array}{lll}
{[\tilde{3}]} & {[\tilde{1}]} & {[\tilde{4}]} \\
{\left[\tilde{f}_{p}^{\prime}\right]} & {[\tilde{1}]} & {\left[\tilde{f}_{p_{A}}\right]} \\
{\left[\tilde{f}^{\prime}\right]} & {\left[\tilde{f}_{2}\right]} & {\left[\tilde{f}_{A}\right]}
\end{array}\right)
$$

in which the left columns and top rows are the same. The analytical expression for this product can be obtained using Eq. (23) from Ref. [76], which gives

$$
\begin{aligned}
& \left(\begin{array}{lll}
{[\tilde{3}]} & {[\tilde{1}]} & {[\tilde{4}]} \\
{\left[\tilde{f}_{p}^{\prime}\right]} & 0 & {\left[\tilde{f}_{p}^{\prime}\right]} \\
{\left[\tilde{f}^{\prime}\right]} & {[\tilde{1}]} & {\left[\tilde{f}_{A-1}\right]}
\end{array}\right)\left\langle\left[\tilde{f}^{\prime}\right] S^{\prime} T^{\prime},[\tilde{1}] \frac{1}{2} \frac{1}{2} \mid\left[\tilde{f}_{A-1}\right] S_{A-1} T_{A-1}\right\rangle \\
& \quad=\frac{1}{2} \frac{\hat{S}^{\prime} \hat{T}^{\prime}}{\hat{S}_{A-1} \hat{T}_{A-1}}\left\langle[\tilde{3}] \frac{1}{2} \frac{1}{2},\left[\tilde{f}_{A-1}\right] S_{A-1} T_{A-1} \mid\left[\tilde{f}^{\prime}\right] S^{\prime} T^{\prime}\right\rangle,
\end{aligned}
$$

where $\hat{i}=\sqrt{2 i+1}$, and

$$
\begin{aligned}
& \left(\begin{array}{lll}
{[\tilde{3}]} & {[\tilde{1}]} & {[\tilde{4}]} \\
{\left[\tilde{f}_{p}^{\prime}\right]} & {[\tilde{1}]} & {\left[\tilde{f}_{p_{A}}\right]} \\
{\left[\tilde{f}^{\prime}\right]} & {\left[\tilde{f}_{2}\right]} & {\left[\tilde{f}_{A}\right]}
\end{array}\right)\left\langle\left[\tilde{f}^{\prime}\right] S^{\prime} T^{\prime},\left[\tilde{f}_{2}\right] S_{2} T_{2} \mid\left[\tilde{f}_{A}\right] S_{A} T_{A}\right\rangle \\
& =(-)^{S_{A}-T_{A}+S_{2}-T_{2}} \frac{1}{2} \hat{S}_{2} \hat{T}_{2} \hat{S}^{\prime} \hat{T}^{\prime} \sum_{S_{A-1} T_{A-1}}\left\{\begin{array}{lll}
S_{A-1} & \frac{1}{2} & S_{A} \\
S_{2} & S^{\prime} & \frac{1}{2}
\end{array}\right\} \\
& \quad \times\left\{\begin{array}{lll}
T_{A-1} & \frac{1}{2} & T_{A} \\
T_{2} & T^{\prime} & \frac{1}{2}
\end{array}\right\}\left\langle\left[\tilde{f}_{p_{A-1}}\right] S_{A-1} T_{A-1},[\tilde{1}] \frac{1}{2} \frac{1}{2} \mid\left[\tilde{f}_{p_{A}}\right] S_{A} T_{A}\right\rangle \\
& \quad \times\langle\tilde{3}] \frac{1}{2} \frac{1}{2},\left[\tilde{f}_{A-1}\right] S_{A-1} T_{A-1}\left|\left[\tilde{f}^{\prime}\right] S^{\prime} T^{\prime}\right\rangle .
\end{aligned}
$$

The objects in angular brackets in Eqs. (A9) and (A10) are the Clebsch-Gordan coefficients for the SU(4) group. The objects in figure brackets in Eq. (A10) are the usual $6 j$ symbols. Eliminating $\left\langle[\tilde{3}] \frac{1}{2} \frac{1}{2},\left[\tilde{f}_{A-1}\right] S_{A-1} T_{A-1} \mid\left[\tilde{f}^{\prime}\right] S^{\prime} T^{\prime}\right\rangle$ from Eq. (A10) using the substitution that follows from Eq. (A9) we get

$$
\begin{aligned}
& \left(\begin{array}{lll}
{[\tilde{3}]} & {[\tilde{1}]} & {[\tilde{4}]} \\
{\left[\tilde{f}_{p}^{\prime}\right]} & 0 & {\left[\tilde{f}_{p}^{\prime}\right]} \\
{\left[\tilde{f}^{\prime}\right]} & {[\tilde{1}]} & {\left[\tilde{f}_{A-1}\right]}
\end{array}\right)\left(\begin{array}{lll}
{[\tilde{3}]} & {[\tilde{1}]} & {[\tilde{4}]} \\
{\left[\tilde{f}_{p}^{\prime}\right]} & {[\tilde{1}]} & {\left[\tilde{f}_{p_{A}}\right]} \\
{\left[\tilde{f}^{\prime}\right]} & {\left[\tilde{f}_{2}\right]} & {\left[\tilde{f}_{A}\right]}
\end{array}\right) \\
& =\frac{N\left(\left[\tilde{f}^{\prime}\right]\right)}{4 N\left(\left[\tilde{f}_{A-1}\right]\right)} \frac{(-)^{S_{A}-T_{A}+S_{2}-T_{2}} \hat{S}_{2} \hat{T}_{2}}{\left\langle\left[\tilde{f}^{\prime}\right] S^{\prime} T^{\prime},\left[\tilde{f}_{2}\right] S_{2} T_{2} \mid\left[\tilde{f}_{A}\right] S_{A} T_{A}\right\rangle} \\
& \times \sum_{S_{A-1} T_{A-1}}\left\{\begin{array}{lll}
S_{A-1} & \frac{1}{2} & S_{A} \\
S_{2} & S^{\prime} & \frac{1}{2}
\end{array}\right\}\left\{\begin{array}{lll}
T_{A-1} & \frac{1}{2} & T_{A} \\
T_{2} & T^{\prime} & \frac{1}{2}
\end{array}\right\} \\
& \times\left\langle\left[\tilde{f}^{\prime}\right] S^{\prime} T^{\prime},[\tilde{1}] \frac{1}{2} \frac{1}{2} \mid\left[\tilde{f}_{A-1}\right] S_{A-1} T_{A-1}\right\rangle \\
& \times\left\langle\left[\tilde{f}_{A-1}\right] S_{A-1} T_{A-1},[\tilde{1}] \frac{1}{2} \frac{1}{2} \mid\left[\tilde{f}_{p_{A}}\right] S_{A} T_{A}\right\rangle,
\end{aligned}
$$

where

$$
\left(\begin{array}{lll}
{[\tilde{3}]} & {[\tilde{1}]} & {[\tilde{4}]} \\
{\left[\tilde{f}_{p}^{\prime}\right]} & 0 & {\left[\tilde{f}_{p}^{\prime}\right]} \\
{\left[\tilde{f}^{\prime}\right]} & {[\tilde{1}]} & {\left[\tilde{f}_{A-1}\right]}
\end{array}\right)^{2}=\frac{N\left(\left[\tilde{f}^{\prime}\right]\right)}{4 N\left[\tilde{f}_{A-1}\right]}
$$

was used. Thus both the absolute value and the sign of the product of two $9 j$ symbols are uniquely determined by known spin-isospin CFPs.
[1] V. R. Pandharipande, I. Sick, and P. K. A. deWitt Huberts, Rev. Mod. Phys. 69, 981 (1997).

[2] G. J. Kramer, H. P. Blok, and L. Lapikás, Nucl. Phys. A 679, 267 (2001).

[3] J. Lee et al., Phys. Rev. C 73, 044608 (2006).

[4] B. A. Brown, P. G. Hansen, B. M. Sherrill, and J. A. Tostevin, Phys. Rev. C 65, 061601 (2002).

[5] A. Gade et al., Phys. Rev. C 77, 044306 (2008).

[6] E. K. Warburton, B. A. Brown, and D. J. Millener, Phys. Lett. B 293, 7 (1992).

[7] L. Lapikás, J. Wesseling, and R. B. Wiringa, Phys. Rev. Lett. 82, 4404 (1999).
[8] C. Bisconti, F. A. de Saavedra, and G. Co, Phys. Rev. C 75, 054302 (2007).

[9] C. Barbieri, Phys. Rev. Lett. 103, 202502 (2009).

[10] C. Barbieri, arXiv:0909.0336v2 [nucl-th].

[11] N. K. Timofeyuk, Phys. Rev. Lett. 103, 242501 (2009).

[12] G. R. Satchler, Direct Nuclear Reactions (Oxford University Press, New York, 1983).

[13] H. Feldemeier et al., Nucl. Phys. A 632, 61 (1998).

[14] P. Descouvemont, Phys. Rev. C 70, 065802 (2004).

[15] K. Bennaceur, F. Nowacki, J. Okolowicz, and N. Ploszajczak, Nucl. Phys. A 651, 289 (1999).

[16] W. T. Pinkston and G. R. Satchler, Nucl. Phys. 72, 641 (1965). 
[17] R. J. Philpott, W. T. Pinkston, and G. R. Satchler, Nucl. Phys. A 119, 241 (1968).

[18] N. K. Timofeyuk, Ph.D. thesis, INP, Tashkent, 1990.

[19] A. M. Mukhamedzhanov et al., Sov. J. Nucl. Phys. 52, 452 (1990).

[20] L. D. Blokhintsev, I. Borbely, and E. I. Dolinskii, Sov. J. Part. Nuclei 8, 485 (1977).

[21] A. M. Mukhamedzhanov and N. K. Timofeyuk, Sov. J. Nucl. Phys. 51, 431 (1990) [Yad. Fiz. 51, 679 (1990)].

[22] R. B. Begzhanov et al., Bull. Acad. Sci. USSR, Phys. Ser. 49, 117 (1985); S. A. Goncharov et al., Yad. Fiz. 44, 303 (1986); G. S. Valiev et al., Bull. Acad. Sci. USSR, Phys. Ser. 51, 124 (1987).

[23] G. Bertsch, J. Borysowicz, H. McManus, and W. G. Love, Nucl. Phys. A 284, 399 (1977).

[24] N. K. Timofeyuk, Nucl. Phys. A 632, 19 (1998).

[25] J. P. Elliott, A. D. Jackson, H. A. Mavromatis, E. A. Sanderson, and B. Singh, Nucl. Phys. A 121, 241 (1968).

[26] N. Anantaraman, H. Toki, and G. F. Bertsch, Nucl. Phys. A 398, 269 (1983).

[27] D. Gogny, P. Pires, and R. De Tourreil, Phys. Lett. B 32, 591 (1970).

[28] R. B. Wiringa, V. G. J. Stoks, and R. Schiavilla, Phys. Rev. C 51, 38 (1995).

[29] I. Borbély, W. Grüebler, V. König, P. A. Schmelzbach, and A. M. Mukhamedzhanov, Phys. Lett. B 160, 17 (1985).

[30] The normalized Jacobi coordinates are defined here as $\boldsymbol{\xi}_{i}=$ $\sqrt{i /(i+1)}\left(\sum_{j=1}^{i} \boldsymbol{r}_{j} / i-\boldsymbol{r}_{i+1}\right)$.

[31] A. Kievsky et al., Phys. Lett. B 406, 292 (1997).

[32] Three- and four-body calculations of Refs. [31,38] use a different definition of Jacobi coordinates. As a result, the overlaps involving these wave functions must be renormalized to be consistent with the definition adopted in standard reactions codes. Also, the definition of ANCs in Refs. [35,38] is different from the one given by Eq. (6) so that the ab initio ANCs shown here are renormalized.

[33] I. Borbély and A. M. Mukhamedzhanov, in Proceedings of the 34 Conference on Nuclear Spectroscopy and Structure, Alma-Ata, USSR, 1984, p. 413 (unpublished).

[34] S. V. Artemov, E. A. Zaparov, and G. K. Nie, Bull. Rus. Acad. Sci., Phys. 67, 1741 (2003).

[35] A. M. Mukhamedzhanov, R. E. Tribble, and N. K. Timofeyuk Phys. Rev. C 51, 3472 (1995).

[36] S. V. Artemov, A. G. Bajajin, S. B. Igamov, G. K. Nie, and R. Yarmukhamedov, Phys. At. Nucl. 71, 998 (2008); Yad. Fiz. 71, 1025 (2008).

[37] N. K. Timofeyuk, R. C. Johnson, and A. M. Mukhamedzhanov, Phys. Rev. Lett. 91, 232501 (2003).

[38] M. Viviani, A. Kievsky, and S. Rosati, Phys. Rev. C 71, 024006 (2005).

[39] C. W. De Jager, H. De Vries, and C. De Vries, At. Data Nucl. Data Tables 14, 479 (1974).

[40] M. Leuschner et al., Phys. Rev. C 49, 955 (1994).
[41] A. M. Mukhamedzhanov et al., Phys. Rev. C 78, 015804 (2008).

[42] A. N. Boyarkina, Structure of p-Shell Nuclei (Moscow State University, Moscow, 1973) [in Russian].

[43] L. Rosenfeld, Nuclear Forces (North Holland, Amsterdam, 1948).

[44] S. Cohen and D. Kurath, Nucl. Phys. 73, 1 (1965).

[45] D. J. Millener, in Topics in Strangeness Nuclear Physics, Lecture Notes in Nuclear Physics, Vol. 724, edited by P. Bydzovsky, A. Gal, and J. Mares (Springer, New York, 2007), p. 31; arXiv:0902.2142 [nucl-th].

[46] R. C. Runkle, A. E. Champagne, C. Angulo, C. Fox, C. Iliadis, R. Longland, and J. Pollanen, Phys. Rev. Lett. 94, 082503 (2005).

[47] P. F. Bertone, A. E. Champagne, M. Boswell, C. Iliadis, S. E. Hale, V. Y. Hansper, and D. C. Powell, Phys. Rev. C 66, 055804 (2002).

[48] A. M. Mukhamedzhanov et al., Phys. Rev. C 67, 065804 (2003).

[49] P. Bem et al., Phys. Rev. C 62, 024320 (2000).

[50] L. Trache et al., Phys. Rev. C 58, 2715 (1998).

[51] I. R. Gulamov, A. M. Mukhamedzhanov, and G. K. Nie, Phys. At. Nucl. 58, 1689 (1995).

[52] X. Tang et al., Phys. Rev. C 69, 055807 (2004).

[53] Z. H. Li et al., Phys. Rev. C 74, 035801 (2006).

[54] H. P. Gubler, G. R. Plattner, I. Sick, A. Traber, and W. Weiss, Nucl. Phys. A 284, 114 (1977).

[55] A. Banu et al., Phys. Rev. C 79, 025805 (2009).

[56] B. Guo, Z. H. Li, W. P. Liu, and X. X. Bai, J. Phys. G 34, 103 (2007).

[57] Z. H. Liu et al., Phys. Rev. C 64, 034312 (2001).

[58] X. Tang et al., Phys. Rev. C 67, 015804 (2003).

[59] A. M. Mukhamedzhanov et al., Phys. Rev. C 56, 1302 (1997).

[60] B. Guo et al., Nucl. Phys. A 761, 162 (2005).

[61] L. Trache, F. Carstoiu, A. M. Mukhamedzhanov, and R. E. Tribble, Phys. Rev. C 66, 035801 (2002).

[62] D. Beaumel et al., Phys. Lett. B 514, 226 (2001).

[63] J. Enders et al., Phys. Rev. C 67, 064301 (2003).

[64] L. Trache et al., Phys. Rev. C 67, 062801(R) (2003).

[65] A. Azhari et al., Phys. Rev. Lett. 82, 3960 (1999).

[66] A. Azhari et al., Phys. Rev. C 60, 055803 (1999).

[67] R. Kanungo et al., Phys. Lett. B 660, 26 (2008).

[68] A. H. Wuosmaa et al., Phys. Rev. C 78, 041302(R) (2008).

[69] R. B. Wiringa, [http://www.phy.anl.gov/theory/research/overlap].

[70] N. Michel, W. Nazarewicz, and M. Ploszajczak, Phys. Rev. C 75, 031301 (R) (2007).

[71] N. K. Timofeyuk, L. D. Blokhintsev, and J. A. Tostevin, Phys. Rev. C 68, 021601 (R) (2003).

[72] N. K. Timofeyuk, I. J. Thompson, and J. A. Tostevin, J. Phys. Conf. Ser. 111, 012034 (2008).

[73] Yu. F. Smirnov and Yu. M. Tchuvil'sky, Phys. Rev. C 15, 84 (1977).

[74] J. D. Vergados, Nucl. Phys. A 111, 681 (1968).

[75] K. T. Hecht and S. C. Pang, J. Math. Phys. 10, 1571 (1969).

[76] V. I. Kukulin, Yu. F. Smirnov, and L. Majling, Nucl. Phys. A 103, 681 (1967).

[77] M. Moshinsky and Yu. F. Smirnov, The Harmonic Oscillator in Modern Physics (Harwood Academic, Amsterdam, 1996). 